\title{
STRUCTURAL STABILITY FOR BANG-SINGULAR-BANG EXTREMALS IN THE MINIMUM TIME PROBLEM *
}

\author{
Laura Poggiolini and Gianna Stefani ${ }^{\dagger}$
}

\begin{abstract}
In this paper we study the structural stability of a bang-singular-bang extremal in the minimum time problem between fixed points. The dynamics is single-input and control-affine.

On the nominal problem $(r=0)$, we assume the coercivity of a suitable second variation along the singular arc and regularity both of the bang arcs and of the junction points, thus obtaining the strict strong local optimality for the given bangsingular-bang extremal trajectory. Moreover, as in the classically studied regular cases, we assume a suitable controllability property, which grants the uniqueness of the adjoint covector.

Under these assumptions we prove that, for any sufficiently small $r$, there is a bang-singular-bang extremal trajectory which is a strict strong local optimiser for the $r$-problem. A uniqueness result in a neighbourhood of the graph of the nominal extremal pair is also obtained.

The results are proven via the Hamiltonian approach to optimal control and by taking advantage of the implicit function theorem, so that a sensitivity analysis could also be carried out.
\end{abstract}

Keywords: Hamiltonian methods, second variation, structural stability.

\section{Introduction}

Since in practical optimisation problems the values of the data usually are not known exactly and/or are subject to disturbances, stability and sensitivity analysis constitute a crucial element of the so-called post-optimisation analysis, which helps to evaluate the practical usefulness of the obtained results.

Here we study the structural stability of a bang-singular-bang extremal in the minimum time problem where the dynamics is single-input and control-affine. The paper is based on the Hamiltonian approach which is used both in the optimality and in the stability results.

We point out that, as in the classically studied regular cases (see [11, 12, 13]), the assumptions on the nominal problem are the ones which give optimality, see [16, 17], together with a controllability assumption which grants the uniqueness of the adjoint covector.

\footnotetext{
*This work was partially supported by PRIN 200894484E_002, Controllo Nonlineare: metodi geometrici e applicazioni

${ }^{\dagger}$ Dipartimento di Sistemi e Informatica - Università degli Studi di Firenze, Italy (laura.poggiolini@unifi.it, gianna.stefani@unifi.it).
} 
The parameter-dependent minimum time problem $\left(\mathbf{P}_{\mathbf{r}}\right)$ we study is given by

$$
\begin{aligned}
& \dot{\xi}^{r}(t)=f_{0}^{r}(\xi(t))+u(t) f_{1}^{r}(\xi(t)) \\
& u(t) \in[-1,1]
\end{aligned}
$$

and is constrained to

$$
\xi^{r}(0)=a^{r}, \quad \xi^{r}(T)=b^{r},
$$

where $a^{r}$ and $b^{r}$ are two given points. The parameter $r$ is in $\mathbb{R}^{m}$, the state space is $\mathbb{R}^{n}$ (but the result can be easily generalised to the case when the state space is a smooth finite dimensional manifold) and all the data are assumed to be smooth, say $C^{\infty}$.

We study two different kinds of strong local optimality of a triplet $\left(T^{r}, \xi^{r}, u^{r}\right)$ which is admissible for $\left(\mathbf{P}_{\mathbf{r}}\right)$ according to the following definitions

Definition 1.1. The trajectory $\xi^{r}:\left[0, T^{r}\right] \rightarrow \mathbb{R}^{n}$ is a (time, state)-local minimiser of $\left(\mathbf{P}_{\mathbf{r}}\right)$ if there is a neighbourhood $\widetilde{\mathcal{U}}$ of its graph in $\mathbb{R} \times \mathbb{R}^{n}$ and $\varepsilon>0$ such that $\xi^{r}$ is a minimiser among the admissible trajectories whose graphs are in $\widetilde{\mathcal{U}}$ and whose final time is greater than $T^{r}-\varepsilon$, independently of the values of the associated controls.

We point out that this kind of optimality is local both with respect to time and space. A stronger version of strong local optimality is the so-called state-local optimality which is defined as follows:

Definition 1.2. The trajectory $\xi^{r}$ is a state-local minimiser of $\left(\mathbf{P}_{\mathbf{r}}\right)$ if there is a neighbourhood $\mathcal{U}$ of its range in $\mathbb{R}^{n}$ such that $\xi^{r}$ is a minimiser among the admissible trajectories whose range is in $\mathcal{U}$, independently of the values of the associated controls.

For the nominal problem $(r=0)$, we assume the coercivity of a suitable second variation along the singular arc and regularity both of the bang arcs and of the junction points, thus obtaining the strict state local optimality for the given bang-singular-bang extremal, and a suitable controllability assumption along the singular arc only, see Section 2 ,

Under these assumptions we shall prove that, for any sufficiently small $r \in \mathbb{R}^{m}$, there is a bang-singular-bang extremal trajectory $\xi^{r}$ which is a strict strong local optimiser for problem $\left(\mathbf{P}_{\mathbf{r}}\right)$. Moreover, if $\mu^{r}$ is the costate associated to $\xi^{r}$, then there exists a neighbourhood $\mathcal{V}$ of the graph of the nominal pair $\widehat{\lambda}=(\widehat{\mu}, \widehat{\xi})$ such that $\left(\mu^{r}, \xi^{r}\right)$ is the only extremal pair of $\left(\mathbf{P}_{\mathbf{r}}\right)$ whose graph is in $\mathcal{V}$.

The results are proven via the Hamiltonian approach to optimal control and by taking advantage of the implicit function theorem. Thus the trajectory $\xi^{r}$ and its switching times depend smoothly on the parameter $r$, so that a sensitivity analysis could also be carried out.

For the regular cases we refer to [11, 12, 13] and the references therein. For control affine dynamics we mention [6, 8, 14, 15] where bang-bang extremals for the nominal problem are considered. Bang-singular-bang extremals for the Mayer problem are studied also in [7, 5] where the author, under suitable assumptions, shows that if the perturbed problem has an extremal which is some sense near the reference one, then this extremal has the same bang-singular-bang structure. 
We assume we are given a reference triplet $(\widehat{T}, \widehat{\xi}, \widehat{u})$ which is a normal bang-singularbang Pontryagin extremal for the nominal problem $\left(\mathbf{P}_{\mathbf{0}}\right)$ that is $\widehat{u}$ has the following structure

$$
\begin{array}{ll}
\widehat{u}(t) \equiv u_{1} \in\{-1,1\} & \forall t \in\left[0, \widehat{\tau}_{1}\right), \\
\widehat{u}(t) \in(-1,1) & \forall t \in\left(\widehat{\tau}_{1}, \widehat{\tau}_{2}\right), \\
\widehat{u}(t) \equiv u_{2} \in\{-1,1\} & \forall t \in\left(\widehat{\tau}_{2}, \widehat{T}\right] .
\end{array}
$$

so that the reference vector field driving the nominal system is given by

$$
\widehat{f_{t}}= \begin{cases}h_{1}:=f_{0}+u_{1} f_{1} & \text { if } t \in\left[0, \widehat{\tau}_{1}\right) \\ f_{0}+\widehat{u}(t) f_{1} & \text { if } t \in\left(\widehat{\tau}_{1}, \widehat{\tau}_{2}\right) \\ h_{2}:=f_{0}+u_{2} f_{1} & \text { if } t \in\left(\widehat{\tau}_{2}, \widehat{T}\right]\end{cases}
$$

We shall refer to $\widehat{\tau}_{1}, \widehat{\tau}_{2}$ as to the switching times of the reference control $\widehat{u}$.

The plan of the paper is as follows: we conclude this section by giving the fundamental notation. In Section 2 we state the assumptions on the nominal problem; the regularity assumptions are stated in Section 2.1 while the coercivity and the controllability assumptions are stated in Sections 2.2 and 2.3. In Section 3 we give the main results and an example. Finally in Section 4 we give all the proofs of the main results.

\subsection{Notation}

In this paper we use some basic element of the theory of symplectic manifolds for the cotangent bundle $T^{*} \mathbb{R}^{n}=\left(\mathbb{R}^{n}\right)^{*} \times \mathbb{R}^{n}$. For a general introduction see [2], for specific application to Control Theory see e.g. [1]. Let us recall some basic facts and let us introduce some specific notations.

We denote by $\pi: \ell=(p, q) \in T^{*} \mathbb{R}^{n} \mapsto q \in \mathbb{R}^{n}$ the canonical projection. If $V \subset \mathbb{R}^{n}$ we denote as $V^{\perp} \subset\left(\mathbb{R}^{n}\right)^{*}$ its orthogonal space. The symbol $s$ denotes the canonical Liouville one-form on $T^{*} \mathbb{R}^{n}: \quad s:=\sum_{i=1}^{n} p^{i} \mathrm{~d} q_{i}$. The associated canonical symplectic two-form $\boldsymbol{\sigma}=\mathrm{d} \boldsymbol{s}$ allows one to associate to any, possibly time-dependent, smooth Hamiltonian $H_{t}: T^{*} \mathbb{R}^{n} \rightarrow \mathbb{R}$, a Hamiltonian vector field $\vec{H}_{t}$, by

$$
\boldsymbol{\sigma}\left(v, \vec{H}_{t}(\ell)\right)=\left\langle\mathrm{d} H_{t}(\ell), v\right\rangle, \quad \forall v \in T_{\ell} T^{*} \mathbb{R}^{n} .
$$

In coordinates

$$
\vec{H}_{t}(\ell)\left(-\left.\frac{\partial H_{t}}{\partial q}\right|_{\ell},\left.\frac{\partial H_{t}}{\partial p}\right|_{\ell}\right), \quad \forall \ell=(p, q) \in T^{*} \mathbb{R}^{n} .
$$

In this paper the switching time $\widehat{\tau}_{1}$ plays a special role, hence we consider all the flows as starting at time $\widehat{\tau}_{1}$. We denote the flow of $\vec{H}_{t}$ from time $\widehat{\tau}_{1}$ to time $t$ by

$$
\mathcal{H}:(t, \ell) \mapsto \mathcal{H}(t, \ell)=\mathcal{H}_{t}(\ell) .
$$

We keep these notation throughout the paper, namely the overhead arrow denotes the vector field associated to a Hamiltonian and the script letter denotes its flow from time $\widehat{\tau}_{1}$, unless otherwise stated. 
Finally recall that any vector field $f$ on $\mathbb{R}^{n}$ defines, by lifting to the cotangent bundle, a Hamiltonian

$$
F: \ell=(p, q) \in T^{*} \mathbb{R}^{n} \mapsto\langle p, f(q)\rangle \in \mathbb{R} .
$$

We denote by $F_{0}^{r}, F_{1}^{r}, H_{1}^{r}, H_{2}^{r}$, the Hamiltonians associated to $f_{0}^{r}, f_{1}^{r}, h_{1}^{r}, h_{2}^{r}$, respectively and by

$$
F_{i_{1} i_{2} \ldots i_{k}}^{r}:=\left\{F_{i_{1}}^{r},\left\{\ldots\left\{F_{i_{k-1}}^{r}, F_{i_{k}}^{r}\right\} \ldots\right\}, \quad i_{1}, \ldots, i_{k} \in\{0,1\}\right.
$$

the Hamiltonian associated to $f_{i_{1} i_{2} \ldots i_{k}}^{r}:=\left[f_{i_{1}}^{r},\left[\ldots\left[f_{i_{k-1}}^{r}, f_{i_{k}}^{r}\right] \ldots\right]\right.$, where $\{\cdot, \cdot\}$ denotes the Poisson parentheses between Hamiltonians and $[\cdot, \cdot]$ denotes the Lie brackets between vector fields.

The flow from time $\widehat{\tau}_{1}$ of the reference vector field $\widehat{f}_{t}$ is a map defined in a neighbourhood of the point $\widehat{x}_{1}:=\widehat{\xi}\left(\widehat{\tau}_{1}\right)$. We denote it as $\widehat{S}_{t}: \mathbb{R}^{n} \rightarrow \mathbb{R}^{n}, \quad t \in[0, \widehat{T}]$ while

$$
\widehat{F_{t}}= \begin{cases}H_{1} & \text { if } t \in\left[0, \widehat{\tau}_{1}\right) \\ F_{0}+\widehat{u}(t) F_{1} & \text { if } t \in\left(\widehat{\tau}_{1}, \widehat{\tau}_{2}\right) \\ H_{2} & \text { if } t \in\left(\widehat{\tau}_{2}, \widehat{T}\right]\end{cases}
$$

denotes the time-dependent reference Hamiltonian obtained lifting $\widehat{f}_{t}$.

Moreover we define $H^{\max , r}$ to be the continuous maximised Hamiltonian associated to the control system (1)-(2), i.e.

$$
H^{\max , r}: \ell \mapsto \max _{u \in[-1,1]}\left\{F_{0}^{r}(\ell)+u F_{1}^{r}(\ell)\right\}
$$

To facilitate reading, when $r=0$ we omit the parameter, i.e. we write $f_{0}$ instead of $f_{0}^{0}$, $f_{1}$ instead of $f_{1}^{0}, H^{\max }$ instead of $H^{\max , 0}$ and so on.

Also we use the following notation from differential geometry: $f \cdot \alpha$ is the Lie derivative of a function $\alpha$ with respect to the vector field $f$. Moreover, if $G$ is a $C^{1}$ map from a manifold $X$ in a manifold $Y$, we denote its tangent map at a point $x \in X$ as $G_{*}$, if the point $x$ is clear from the context.

\section{Assumptions on the nominal problem}

In this section we state the assumptions on the nominal extremal. Besides Pontryagin Maximum Principle, we state the assumptions which ensure strong local optimality of the reference trajectory, see [17]: regularity assumptions on the bang arcs and on the junction points and a coercivity assumption of a suitable second variation on the singular arc. We are also making one further assumption, i.e. controllability along the singular arc or, equivalently the uniqueness of the adjoint covector.

\subsection{Pontryagin Maximum Principle and Regularity Assumptions}

In this section we recall the first order optimality condition which the reference triplet $(\widehat{T}, \widehat{\xi}, \widehat{u})$ must satisfy.

We call extremal pair of $\left(\mathbf{P}_{\mathbf{0}}\right)$ any curve in the cotangent bundle which satisfies PMP and extremal trajectory of $\left(\mathbf{P}_{\mathbf{0}}\right)$ its projection on the state space. Here we ask for the 
reference trajectory to be a normal extremal trajectory, i.e. we assume that the triplet $(\widehat{T}, \widehat{\xi}, \widehat{u})$ satisfies the following

Assumption 2.1 (Normal PMP). There exists a solution $\widehat{\lambda}=(\widehat{\mu}, \widehat{\xi}):[0, \widehat{T}] \rightarrow T^{*} \mathbb{R}^{n}$ of the Hamiltonian system

$$
\dot{\lambda}(t)=\vec{F}_{t} \circ \lambda(t)
$$

such that

$$
\left\langle\widehat{\mu}(t), \widehat{f}_{t} \circ \widehat{\xi}(t)\right\rangle=\widehat{F}_{t} \circ \widehat{\lambda}(t)=H^{\max } \circ \widehat{\lambda}(t)=1 \quad \text { a.e. } t \in[0, \widehat{T}] .
$$

$\widehat{\mu}$ is called nominal adjoint covector and satisfies the adjoint equation

$$
\dot{\hat{\mu}}(t)=-\frac{\partial \widehat{F}_{t}}{\partial q}(\widehat{\mu}(t), \widehat{\xi}(t))
$$

We denote the initial point, the junction points between the bang and the singular arcs and the final point of $\widehat{\lambda}$ as

$$
\begin{array}{ll}
\widehat{\ell}_{0}=\left(\widehat{\mu}_{0}, \widehat{x}_{0}\right):=\widehat{\lambda}(0), & \widehat{\ell}_{1}=\left(\widehat{\mu}_{1}, \widehat{x}_{1}\right):=\widehat{\lambda}\left(\widehat{\tau}_{1}\right), \\
\widehat{\ell}_{2}=\left(\widehat{\mu}_{2}, \widehat{x}_{2}\right):=\widehat{\lambda}\left(\widehat{\tau}_{2}\right), \quad \widehat{\ell}_{f}=\left(\widehat{\mu}_{f}, \widehat{x}_{f}\right):=\widehat{\lambda}(\widehat{T}),
\end{array}
$$

respectively. Because of the structure of the reference control $\widehat{u}$, as defined by equations (4), PMP implies

$$
\begin{array}{ll}
u_{1} F_{1} \circ \widehat{\lambda}(t) \geq 0 & t \in\left[0, \widehat{\tau}_{1}\right), \\
F_{1} \circ \widehat{\lambda}(t)=0 & t \in\left[\widehat{\tau}_{1}, \widehat{\tau}_{2}\right], \\
u_{2} F_{1} \circ \widehat{\lambda}(t) \geq 0 & t \in\left(\widehat{\tau}_{2}, \widehat{T}\right] .
\end{array}
$$

As a consequence, see [17], one gets

$$
\begin{array}{ll}
F_{01} \circ \widehat{\lambda}(t) \equiv 0 \quad t \in\left[\widehat{\tau}_{1}, \widehat{\tau}_{2}\right], & \left(F_{001}+\widehat{u}(t) F_{101}\right) \circ \widehat{\lambda}(t)=0 \quad t \in\left(\widehat{\tau}_{1}, \widehat{\tau}_{2}\right), \\
u_{1}\left(F_{001}+u_{1} F_{101}\right)\left(\widehat{\ell}_{1}\right) \geq 0, & u_{2}\left(F_{001}+u_{2} F_{101}\right)\left(\widehat{\ell}_{2}\right) \geq 0 .
\end{array}
$$

PMP yields the mild inequalities in (6), (8) and (10). We assume the strict inequalities to hold, whenever possible.

Assumption 2.2 (Regularity along the bang arcs).

$$
u_{1} F_{1} \circ \widehat{\lambda}(t)>0 \quad \forall t \in\left[0, \widehat{\tau}_{1}\right), \quad u_{2} F_{1} \circ \widehat{\lambda}(t)>0 \quad \forall t \in\left(\widehat{\tau}_{2}, \widehat{T}\right] .
$$

Assumption 2.3 (Regularity at the junction points).

$$
\left(u_{1} F_{001}+F_{101}\right)\left(\widehat{\ell}_{1}\right)>0, \quad\left(u_{2} F_{001}+F_{101}\right)\left(\widehat{\ell}_{2}\right)>0 .
$$


Another well known necessary condition for the local optimality of a Pontryagin extremal is the generalised Legendre condition (GLC) along the singular arc:

$$
F_{101} \circ \widehat{\lambda}(t) \geq 0 \quad t \in\left[\widehat{\tau}_{1}, \widehat{\tau}_{2}\right],
$$

see for example [1], Corollary 20.18 page 318; for a classical result see [9]. The coercivity assumption stated in the next section implies the Strengthened generalised Legendre condition

$$
F_{101} \circ \widehat{\lambda}(t)>0, \quad t \in\left[\widehat{\tau}_{1}, \widehat{\tau}_{2}\right] .
$$

When (SGLC) holds, a singular extremal is called of the first kind, see e.g. 22.

Remark 2.1. (SGLC) implies that $\widehat{u} \in C^{\infty}\left(\left(\widehat{\tau}_{1}, \widehat{\tau}_{2}\right)\right)$ and that Assumption 2.3 is equivalent to the discontinuity of $\widehat{u}$ at times $\widehat{\tau}_{1}$ and $\widehat{\tau}_{2}$, see [17].

Assumption 2.4 (Uniqueness of the adjoint covector). $\left.\widehat{\lambda}\right|_{\left[\widehat{\tau}_{1}, \widehat{\tau}_{2}\right]}$ is the only adjoint covector associated to $\left.\widehat{\xi}\right|_{\left[\widehat{\tau}_{1}, \widehat{\tau}_{2}\right]}$ for the minimum time problem between $\widehat{\xi}\left(\widehat{\tau}_{1}\right)$ and $\widehat{\xi}\left(\widehat{\tau}_{2}\right)$.

\subsection{Coercivity and controllability assumptions}

System (11) is affine with respect to the control, therefore the standard second variation is completely degenerate. In [17] we transformed the given minimum time problem in a Mayer problem on a fixed time interval and - via a coordinate-free version of Goh's transformation - we obtained a suitable second order approximation on the singular arc, which we call extended second variation.

Proceeding as in Lemma 1 of [18] one can show that the largest sub-space where the extended second variation can be coercive is the one relative to the minimum time problem with fixed end points $\xi\left(\widehat{\tau}_{1}\right)=\widehat{x}_{1}, \xi\left(\widehat{\tau}_{2}\right)=\widehat{x}_{2}$.

We point out that the same assumption, together with Assumptions 2.2 2.3 is sufficient for $\widehat{\xi}$ to be a minimum time trajectory between $\widehat{x}_{0}$ and $\widehat{x}_{f}$, see [17].

For the sake of completeness we write here the above mentioned Mayer problem:

$$
\text { Minimise } \xi^{0}\left(\widehat{\tau}_{2}\right)
$$

subject to

$$
\begin{aligned}
& \dot{\xi}^{0}(s)=u_{0}(s) \\
& \dot{\xi}(s)=u_{0}(s) f_{0}(\xi(s))+u_{0}(s) u(s) f_{1}(\xi(s)) \quad s \in\left[\widehat{\tau}_{1}, \widehat{\tau}_{2}\right] \\
& \left(u_{0}(s), u(s)\right) \in(0,+\infty) \times(-1,1) \\
& \xi^{0}\left(\widehat{\tau}_{1}\right)=\widehat{\tau}_{1}, \quad \xi\left(\widehat{\tau}_{1}\right)=\widehat{x}_{1}, \quad \xi^{0}\left(\widehat{\tau}_{2}\right) \in \mathbb{R}, \quad \xi\left(\widehat{\tau}_{2}\right)=\widehat{x}_{2} .
\end{aligned}
$$

Also, for the sake of future computations we introduce the dragged vector fields at time $\widehat{\tau}_{1}$, along the reference flow, by setting

$$
g_{i, t}(x):=\widehat{S}_{t *}^{-1} f_{i} \circ \widehat{S}_{t}(x), i=0,1, \quad \widehat{g}_{t}:=\widehat{S}_{t *}^{-1} \widehat{f}_{t} \circ \widehat{S}_{t}(x)=g_{0, t}+\widehat{u}(t) g_{1, t},
$$


and we recall that

$$
\dot{g}_{1, t}(x)=\widehat{S}_{t *}^{-1} f_{01} \circ \widehat{S}_{t}(x), \quad \dot{g}_{0, t}(x)=-\widehat{u}(t) \dot{g}_{1, t}(x) .
$$

Since the extremal $\widehat{\lambda}$ is normal, $f_{0}$ and $f_{1}$ are linearly independent at $\widehat{x}_{1}$, so that we may choose local coordinates around $\widehat{x}_{1}$ which simplify computations. Namely, we choose coordinates $y=\left(y_{1}, \ldots, y_{n}\right)$ such that
a. $f_{1}$ is constant: $f_{1} \equiv \frac{\partial}{\partial y_{1}}$,
b. $f_{0}=\frac{\partial}{\partial y_{2}}-y_{1}\left(f_{01}\left(\widehat{x}_{1}\right)+O(y)\right)$.

In such coordinates choose $\beta$ as $\beta(y):=-\sum_{i=2}^{n} \mu_{i} y_{i}$, where $\left(0, \mu_{2}, \ldots, \mu_{n}\right)$ are the coordinates of $\widehat{\mu}_{1}$. We get $\mu_{2}=1, f_{1} \cdot \beta \equiv 0$, and $f_{0} \cdot f_{0} \cdot \beta\left(\widehat{x}_{1}\right)=0$. In these coordinates the extended second variation is thus actually given by the quadratic form

$$
\begin{aligned}
J_{\text {ext }}^{\prime \prime}\left(\varepsilon_{0}, \varepsilon_{1}, w\right)=\frac{1}{2} \int_{\widehat{\tau}_{1}}^{\widehat{\tau}_{2}}\left(w^{2}(t)\left[\dot{g}_{1, t}, g_{1, t}\right] \cdot \beta\left(\widehat{x}_{1}\right)+\right. & \\
& \left.+2 w(t) \zeta(t) \cdot \dot{g}_{1, t} \cdot \beta\left(\widehat{x}_{1}\right)\right) \mathrm{d} t
\end{aligned}
$$

defined on the linear sub-space $\mathcal{W}$ of $\mathbb{R}^{2} \times L^{2}\left(\left[\widehat{\tau}_{1}, \widehat{\tau}_{2}\right], \mathbb{R}\right)$ of the triplets $\left(\varepsilon_{0}, \varepsilon_{1}, w\right)$ such that the linear system

$$
\dot{\zeta}(t)=w(t) \dot{g}_{1, t}\left(\widehat{x}_{1}\right), \quad \zeta\left(\widehat{\tau}_{1}\right)=\varepsilon_{0} f_{0}\left(\widehat{x}_{1}\right)+\varepsilon_{1} f_{1}\left(\widehat{x}_{1}\right), \quad \zeta\left(\widehat{\tau}_{2}\right)=0
$$

admits a solution $\zeta$, see [17.

Assumption 2.5 (Coercivity). The extended second variation for the minimum time problem between fixed end points on the singular arc is coercive. Namely we require that the quadratic form (11) is coercive on the subspace $\mathcal{W}$ of $\mathbb{R}^{2} \times L^{2}\left(\left[\widehat{\tau}_{1}, \widehat{\tau}_{2}\right], \mathbb{R}\right)$ given by the variations $\delta e=\left(\varepsilon_{0}, \varepsilon_{1}, w\right)$ such that system (12) admits a solution.

Remark 2.2. 1. $J_{\text {ext }}^{\prime \prime}$ is a quadratic form defined in the whole space $\mathbb{R}^{2} \times L^{2}\left(\left[\widehat{\tau}_{1}, \widehat{\tau}_{2}\right], \mathbb{R}\right)$, but only its restriction to $\mathcal{W}$ is coordinate free.

2. Notice that

$$
R(t):=\left[\dot{g}_{1, t}, g_{1, t}\right] \cdot \beta\left(\widehat{x}_{1}\right)=F_{101}(\widehat{\lambda}(t))>0 .
$$

3. Under (SGLC) $J_{\text {ext }}^{\prime \prime}$ can be proven to be the standard second variation, along the extremal pair $\left.\hat{\lambda}\right|_{\left[\widehat{\tau}_{1}, \widehat{\tau}_{2}\right]}$ of a nonsingular Mayer problem, see [16] and [20] for more details.

We now exploit Assumption 2.4 in relation to the controllability space (see e.g. [3]) of system (12):

$$
V:=\operatorname{span}\left\{f_{0}\left(\widehat{x}_{1}\right), f_{1}\left(\widehat{x}_{1}\right), \dot{g}_{1, t}\left(\widehat{x}_{1}\right), t \in\left[\widehat{\tau}_{1}, \widehat{\tau}_{2}\right]\right\}
$$


Lemma 2.1. Assumption 2.4 holds if and only if $V=\mathbb{R}^{n}$.

Proof. $V=\mathbb{R}^{n}$ implies Assumption 2.4. Assume by contradiction that there exists a different adjoint covector $\mu(t)=\widehat{\mu}(t)+\omega(t)=\left(\widehat{\mu}_{1}+\omega_{1}\right) \widehat{S}_{t *}^{-1}$ with associated multiplier $\pi_{0} \in\{0,1\}$. By (5)

$$
\left\langle\omega_{1}, g_{1, t}\left(\widehat{x}_{1}\right)\right\rangle=0, \quad\left\langle\omega_{1}, g_{0, t}\left(\widehat{x}_{1}\right)\right\rangle=\pi_{0}-1
$$

which, for $\pi_{0}=1$ yield

$$
\left\langle\omega_{1}, \dot{g}_{1, t}\left(\widehat{x}_{1}\right)\right\rangle=0, \quad\left\langle\omega_{1}, f_{1}\left(\widehat{x}_{1}\right)\right\rangle=0, \quad\left\langle\omega_{1}, f_{0}\left(\widehat{x}_{1}\right)\right\rangle=0
$$

that is $\omega_{1} \in V^{\perp}=\{0\}$.

If $\pi_{0}=0$, then $\widehat{\mu}_{1}+\omega \in V^{\perp}=\{0\}$ so that the new multiplier is the trivial one, a contradiction.

Assumption 2.4 implies $V=\mathbb{R}^{n}$. Suppose, by contradiction, that there exists $\omega \neq 0$, $\omega \in V^{\perp}$ so that

$$
\begin{aligned}
& \left\langle\omega, g_{1, t}\left(\widehat{x}_{1}\right)\right\rangle=\left\langle\omega, f_{1}\left(\widehat{x}_{1}\right)\right\rangle+\int_{\widehat{\tau}_{1}}^{t}\left\langle\omega, \dot{g}_{1, s}\left(\widehat{x}_{1}\right)\right\rangle \mathrm{d} s=0, \\
& \left\langle\omega, g_{0, t}\left(\widehat{x}_{1}\right)\right\rangle=\left\langle\omega, f_{0}\left(\widehat{x}_{1}\right)+\int_{\widehat{\tau}_{1}}^{t} \dot{g}_{0, s}\left(\widehat{x}_{1}\right) \mathrm{d} s\right\rangle=-\int_{\widehat{\tau}_{1}}^{t} \widehat{u}(s)\left\langle\omega, \dot{g}_{1, s}\left(\widehat{x}_{1}\right)\right\rangle \mathrm{d} s=0 .
\end{aligned}
$$

Therefore $\left(\widehat{\mu}_{1}+\omega\right) \widehat{S}_{t *}^{-1}$ is an adjoint covector along the singular arc of $\widehat{\xi}$ with multiplier $p_{0}=1$, a contradiction.

\subsection{Consequences of coercivity and controllability}

In order to exploit the coercivity assumption we follow 21 and we introduce the Lagrangian subspace and the Hamiltonian associated to the second variation (11), (12), respectively given by

$$
\begin{aligned}
& \begin{aligned}
L & :=\left\{f_{0}\left(\widehat{x}_{1}\right), f_{1}\left(\widehat{x}_{1}\right)\right\}^{\perp} \times \operatorname{span}\left\{f_{0}\left(\widehat{x}_{1}\right), f_{1}\left(\widehat{x}_{1}\right)\right\}= \\
\quad & =\mathbb{R} \overrightarrow{F_{0}}\left(\widehat{\ell}_{1}\right) \oplus \mathbb{R} \vec{F}_{1}\left(\widehat{\ell}_{1}\right) \oplus\left(\left\{f_{0}\left(\widehat{x}_{1}\right), f_{1}\left(\widehat{x}_{1}\right)\right\}^{\perp} \times\{0\}\right),
\end{aligned} \\
& H_{t}^{\prime \prime}(\omega, \delta x):=\frac{-1}{2 R(t)}\left(\left\langle\omega, \dot{g}_{1, t}\left(\widehat{x}_{1}\right)\right\rangle+\delta x \cdot \dot{g}_{1, t} \cdot \beta\left(\widehat{x}_{1}\right)\right)^{2} .
\end{aligned}
$$

Lemma 2.2. Let $\mathcal{H}_{t}^{\prime \prime}:\left(\mathbb{R}^{n}\right)^{*} \times \mathbb{R}^{n} \rightarrow\left(\mathbb{R}^{n}\right)^{*} \times \mathbb{R}^{n}$ be the flow of the Hamiltonian $H_{t}^{\prime \prime}$ defined in (17). Under Assumptions 2.4 and 2.5 the kernel of the linear mapping $\left.\pi_{*} \mathcal{H}_{\widehat{\tau}_{2}}^{\prime \prime}\right|_{L}$ is trivial.

Proof. It is an easy consequence of the results in [21] that the quadratic form $J_{\text {ext }}^{\prime \prime}$ is coercive if and only if for all $(\omega, \delta x) \in L$ and all $t \in\left[\widehat{\tau}_{1}, \widehat{\tau}_{2}\right]$

$$
\pi_{*} \mathcal{H}_{t}^{\prime \prime}(\omega, \delta x)=0 \quad \text { implies } \quad\left\{\begin{array}{l}
\delta x=0 \\
\mathcal{H}_{s}^{\prime \prime}(\omega, 0)=(\omega, 0) \quad \forall s \in\left[\widehat{\tau}_{1}, t\right] .
\end{array}\right.
$$


Ler $\left.(\omega, \delta x) \in \operatorname{ker} \pi_{*} \mathcal{H}_{\widehat{\tau}_{2}}^{\prime \prime}\right|_{L}$. By (18) $\delta x=0, \omega \in\left\{f_{0}\left(\widehat{x}_{1}\right), f_{1}\left(\widehat{x}_{1}\right)\right\}^{\perp}$ and $(\mu(t), \zeta(t)):=$ $\mathcal{H}_{t}^{\prime \prime}(\omega, \delta x)=(\omega, 0)$ for any $t \in\left[\widehat{\tau}_{1}, \widehat{\tau}_{2}\right]$. Since the equations for $(\mu(t), \zeta(t))$ are

$$
\begin{aligned}
& \dot{\mu}(t)=\frac{1}{R(t)}\left(\left\langle\mu(t), \dot{g}_{1, t}\left(\widehat{x}_{1}\right)\right\rangle+\zeta(t) \cdot \dot{g}_{1, t} \cdot \beta\left(\widehat{x}_{1}\right)\right)(\cdot) \cdot \dot{g}_{1, t} \cdot \beta\left(\widehat{x}_{1}\right) \\
& \dot{\zeta}(t)=\frac{-1}{R(t)}\left(\left\langle\mu(t), \dot{g}_{1, t}\left(\widehat{x}_{1}\right)\right\rangle+\zeta(t) \cdot \dot{g}_{1, t} \cdot \beta\left(\widehat{x}_{1}\right)\right) \dot{g}_{1, t}\left(\widehat{x}_{1}\right) .
\end{aligned}
$$

we get $\left\langle\omega, \dot{g}_{t}^{1}\left(\widehat{x}_{1}\right)\right\rangle=0$ for all $t \in\left[\widehat{\tau}_{1}, \widehat{\tau}_{2}\right]$. Thus, Assumption 2.4 yields the claim.

\section{The main results}

In this Section we state the main results of the paper, Theorem 3.1 and 3.2 , which will be proven in the following Section, and provide an example.

Theorem 3.1. Under Assumptions 2.1 2.5, there exists $\rho>0$ such that for any $r$, $\|r\|<\rho$, problem $\left(\mathbf{P}_{\mathbf{r}}\right)$ has a bang-singular-bang strict (time, state)-local optimiser $\xi^{r}$. The switching times and the final time of $\xi^{r}$ depend smoothly on $r$. If $\widehat{\xi}$ is injective, then $\xi^{r}$ is a state-local optimiser of $\left(\mathbf{P}_{\mathbf{r}}\right)$.

First we prove the existence of the bang-singular-bang extremal trajectory $\xi^{r}$, by Hamiltonian methods and the implicit function theorem (see Lemma 4.4). Then the optimality of $\xi^{r}$ is proven by showing - via standard methods of functional analysis that the coercivity and the injectivity conditions are stable under small perturbations of the parameter $r$, see Lemmata 4.5 and 4.6 .

We point out that using the implicit function theorem allows to perform a sensitivity analysis in a standard way; this will be the object of a future analysis.

Furthermore we prove the uniqueness of the extremal pair $\lambda^{r}=\left(\mu^{r}, \xi^{r}\right)$, defined in Theorem 3.1, in a suitable neighbourhood of the graph of the nominal pair $\hat{\lambda}$.

Theorem 3.2. Under Assumptions 2.1 2.5, there exist $\rho>0, \varepsilon>0$ and a neighbourhood $\mathcal{V}$ of the graph of $\hat{\lambda}$ in $\mathbb{R} \times\left(\mathbb{R}^{n}\right)^{*} \times \mathbb{R}^{n}$ such that for any $r,\|r\|<\rho$, the extremal pair $\lambda^{r}$ associated to the local optimiser $\xi^{r}$ of Theorem 3.1 is the only extremal pair of $\left(\mathbf{P}_{\mathbf{r}}\right)$ whose graph is in $\mathcal{V}$ and whose final time is in $[\widehat{T}-\varepsilon, \widehat{T}+\varepsilon]$.

The proof of this result is quite technical and is given in Section 4.4, we conclude this section with an example.

\subsection{Dubins car}

A classical minimum time problem is the so-called Dubins car problem, where the dynamics describes the motion of a car moving in a plane with fixed speed and with bounded, controlled angular velocity. The car has to be steered from a given initial position $\left(x_{0}, y_{0}\right)$ and orientation $\theta_{0}$ to a prescribed final position $\left(x_{f}, y_{f}\right)$ and orientation $\theta_{f}$. Namely the 
problem is

minimise $T$ subject to

$$
\begin{aligned}
& \dot{x}(t)=\cos \theta(t), \quad \dot{y}(t)=\sin \theta(t), \quad \dot{\theta}(t)=u(t), \\
& (x(0), y(0), \theta(0))=\left(x_{0}, y_{0}, \theta_{0}\right), \quad(x(T), y(T), \theta(T))=\left(x_{f}, y_{f}, \theta_{f}\right), \\
& |u(t)| \leq 1 .
\end{aligned}
$$

It can be proven that the only singular control is $u \equiv 0$ and that, if the initial and final positions on the $(x, y)$-plane are sufficiently far, then the optimal trajectory is bang-singularbang, see e.g. [1]. This example fits our assumptions with $f_{0}(x, y, \theta)=(\cos \theta, \sin \theta, 0)^{t}$ and $f_{1}(x, y, \theta)=(0,0,1)^{t}$. An easy computation shows that both Assumptions 2.2 and 2.3 are satisfied. In [16] it is shown that the second variation associated to any singular trajectory between two fixed end points is coercive. Moreover, since $\operatorname{span}\left\{f_{0}, f_{1}, f_{01}\right\}(x, y, \theta)=\mathbb{R}^{3}$ for any $(x, y, \theta) \in \mathbb{R}^{3}$, also Assumption 2.4 is trivially satisfied. Thus the bang-singularbang structure of optimisers in the Dubins car problem is stable under small perturbations of the data of the problem.

When the final orientation $\theta(T)$ is not prescribed, the problem is also quoted as Dodgem car problem, see e.g. [4]. In this case when the initial and final positions on the $(x, y)$-plane are sufficiently far, optimal trajectories are the concatenation of a bang and of a singular arc. The same assumptions stated here for bang-singular-bang extremals yield both optimality and stability of such trajectories, provided that the perturbed final constraint is an integral line of the perturbed controlled vector field. Some preliminary results are in [16] and [18]. Complete proofs will appear in [19].

\section{Proof of the results}

\subsection{Hamiltonian approach}

In this section we describe some properties of the Hamiltonians linked to our system near the singular arc of the reference extremal, for more details see [17].

By (7), (9) and (SGLC), any singular extremal of the first kind of $\left(\mathbf{P}_{\mathbf{0}}\right)$ belongs to the set

$$
\mathcal{S}:=\left\{\ell \in T^{*} M: F_{1}(\ell)=F_{01}(\ell)=0, F_{101}(\ell)>0\right\},
$$

a subset of $\Sigma:=\left\{\ell \in T^{*} M: F_{1}(\ell)=0\right\}$, where the maximised Hamiltonian of $\left(\mathbf{P}_{\mathbf{0}}\right)$, $H^{\max }$, coincides with every Hamiltonian $F_{0}+u F_{1}, u \in \mathbb{R}$.

Notice that $\mathcal{S}$ and $\Sigma$ are independent of the control constraints but, by (21), (9) and Remark 2.1, any singular extremal of problem $\left(\mathbf{P}_{\mathbf{0}}\right)$ is in

$$
\mathcal{S} \cap\left\{\ell \in T^{*} M:\left|\frac{F_{001}}{F_{101}}(\ell)\right|<1\right\} .
$$

The following results are proven in Lemmata 2 and 3 of [17]:

Lemma 4.1. If (SGLC) holds, then there exists a neighbourhood $\mathcal{V}$ of $\mathcal{S}$ in $T^{*} \mathbb{R}^{n}$ where the following statements hold true. 
1. $\Sigma \cap \mathcal{V}$ is a hyper-surface and $\mathcal{S} \cap \mathcal{V}$ is a $(2 n-2)$-dimensional symplectic manifold. Moreover $\Sigma$ separates the regions defined by: $H^{\max }=F_{0}+F_{1}, H^{\max }=F_{0}-F_{1}$.

2. The Hamiltonian vector field $\vec{F}_{1}$ is tangent to $\Sigma$ and transverse to $\mathcal{S}$, while $\overrightarrow{F_{01}}$ is transverse to $\Sigma$.

3. Setting $v:=\frac{-F_{001}}{F_{101}}$ we obtain the Hamiltonian of singular extremals of the first kind

$$
F^{S}:=F_{0}+v F_{1},
$$

i.e. the associated vector field $\overrightarrow{F^{S}}$ is tangent to $\mathcal{S}$ and any singular extremal of the first kind of $\left(\mathbf{P}_{\mathbf{0}}\right)$ is an integral curve of $\overrightarrow{F^{S}}$ contained in $\mathcal{S}$.

4. There exists a non-negative smooth Hamiltonian $\chi: \mathcal{V} \rightarrow[0,+\infty)$ such that

a) $\chi=0, \vec{\chi}=0$ and $\mathrm{D}^{2} \chi=\frac{1}{F_{101}} \mathrm{D} F_{01} \otimes \mathrm{D} F_{01}$ on $\mathcal{S}$;

b) $\overrightarrow{F_{0}}+\vec{\chi}$ is tangent to $\Sigma$.

From now on we shall denote $\Sigma \cap \mathcal{V}$ and $\mathcal{S} \cap \mathcal{V}$ as $\Sigma$ and $\mathcal{S}$, respectively.

Since for the nominal problem (SGLC) holds true in the neighbourhood $\mathcal{V}$ of $\hat{\lambda}$

defined in Lemma 4.1, then possibly restricting $\mathcal{V}$ and for small enough $\|r\|$, (SGLC) holds also for the Hamiltonians $F_{101}^{r}$. Therefore we can define, in $\mathcal{V}$, the Hamiltonians of singular extremals of $\left(\mathbf{P}_{\mathbf{r}}\right)$

$$
F^{S, r}:=F_{0}^{r}-\frac{F_{001}^{r}}{F_{101}^{r}} F_{1}^{r}
$$

In order to prove our main result we are going to use the following result from [17].

Lemma 4.2. If (SGLC) holds, then the Hamiltonian vector field $\overrightarrow{\widehat{H}}_{t}:=\overrightarrow{\widehat{F}}_{t}+\vec{\chi}$ is tangent to $\Sigma$. For any $t \in\left[\widehat{\tau}_{1}, \widehat{\tau}_{2}\right]$ the derivative of its flow $\widehat{\mathcal{H}}_{t}$ satisfies the following properties:

1. $\widehat{\mathcal{H}}_{t *} \vec{F}_{1}\left(\widehat{\ell}_{1}\right)=\vec{F}_{1}(\widehat{\lambda}(t))$ and $\widehat{\mathcal{H}}_{t *} \vec{F}_{0}\left(\widehat{\ell}_{1}\right)=\vec{F}_{0}(\widehat{\lambda}(t))$

2. If $\delta \ell_{S} \in T_{\widehat{\ell}_{1}} \mathcal{S}$ then

$$
\mathcal{F}_{t *}^{S} \delta \ell_{S}=\widehat{\mathcal{H}}_{t *} \delta \ell_{S}+a\left(t, \delta \ell_{S}\right) \vec{F}_{1}(\widehat{\lambda}(t))
$$

where $\mathcal{F}_{t}^{S}$ is the flow of $\overrightarrow{F^{S}}$ and $a\left(t, \delta \ell_{S}\right):=\int_{0}^{t}\left\langle\mathrm{D} v(\widehat{\lambda}(s)), \mathcal{F}_{t *}^{S} \delta \ell_{S}\right\rangle \mathrm{d} s$.

Proof. Claim 1 is proven in Lemma 4 of [17].

Proof of Claim 2: The flow $\mathcal{G}_{t}:=\widehat{\mathcal{H}}_{t}^{-1} \circ \mathcal{F}_{t}^{S}$ is the Hamiltonian flow associated to $G_{t}:=\left((v-\widehat{u}(t)) F_{1}-\chi\right) \circ \widehat{\mathcal{H}}_{t}$. Since $\mathrm{D} G_{t}\left(\widehat{\ell}_{1}\right)=0$, then $\mathcal{G}_{t *}=\widehat{\mathcal{H}}_{t *}^{-1} \mathcal{F}_{t *}^{S}$ is the linear flow associated to the quadratic Hamiltonian

$$
\mathrm{D}^{2} G_{t}\left(\widehat{\ell}_{1}\right)=\left(\mathrm{D} v \otimes \mathrm{D} F_{1}+\mathrm{D} F_{1} \otimes \mathrm{D} v-\frac{1}{F_{101}} \mathrm{D} F_{01} \otimes \mathrm{D} F_{01}\right)(\widehat{\lambda}(t)) \widehat{\mathcal{H}}_{t *} \otimes \widehat{\mathcal{H}}_{t *} .
$$


Set $\gamma(t):=\mathcal{G}_{t *} \delta \ell$. Since $\widehat{\mathcal{H}}_{t *} \mathcal{G}_{t *} \delta \ell=\mathcal{F}_{t *}^{S} \delta \ell \in T_{\widehat{\lambda}(t)} \mathcal{S}$, we obtain, by Claim 1 that $\dot{\gamma}(t)=$ $\left\langle\mathrm{D} v(\widehat{\lambda}(t)), \widehat{\mathcal{F}}_{t *} \delta \ell\right\rangle \vec{F}_{1}\left(\widehat{\ell}_{1}\right)$. Thus $\mathcal{G}_{t *} \delta \ell=\gamma(t)=\delta \ell+\int_{0}^{t}\left\langle\mathrm{D} v(\widehat{\lambda}(s)), \mathcal{F}_{t *}^{S} \delta \ell\right\rangle \mathrm{d} s \vec{F}_{1}\left(\widehat{\ell}_{1}\right)$ which, together with Claim 1, completes the proof.

We end this section by rephrasing Lemma 2.2 in terms of the flow $\widehat{\mathcal{H}}$ defined in Lemma 4.2. This is done adapting the proof of Claim 1 in Lemma 9 of [17].

Corollary 4.3. Under Assumptions 2.4 and 2.5 the kernel of the linear map $\pi_{*} \widehat{\mathcal{H}}_{\widehat{\tau}_{2}}: L \rightarrow$ $\mathbb{R}^{n}$ is trivial.

Proof. $\mathcal{G}_{t}:=\widehat{\mathcal{F}}_{t *}^{-1} \widehat{\mathcal{H}}_{t *}$ is the linear flow associated to the quadratic Hamiltonian

$$
\begin{aligned}
G_{t}(\omega, \delta x) & =\frac{1}{2 R(t)}\left(\mathrm{D} F_{01}(\widehat{\lambda}(t))\left(\omega \widehat{S}_{t *}^{-1}, \widehat{S}_{t *} \delta x\right)\right)^{2} \\
& =\frac{1}{2 R(t)}\left(\left\langle\omega \widehat{S}_{t *}^{-1}, f_{01}(\widehat{\xi}(t))\right\rangle+\left\langle\widehat{\mu}(t), \mathrm{D} f_{01}(\widehat{\xi}(t)) \widehat{S}_{t *} \delta x\right\rangle\right)^{2} \\
& =\frac{1}{2 R(t)}\left(\left\langle\omega, \dot{g}_{1, t}\left(\widehat{x}_{1}\right)\right\rangle-\delta x \cdot \dot{g}_{1, t} \cdot \beta\left(\widehat{x}_{1}\right)\right)^{2} .
\end{aligned}
$$

Consider the linear isomorphism $i:(\omega, \delta x) \mapsto(-\omega, \delta x)$. Then $G_{t}=-H_{t}^{\prime \prime} \circ i$ and $\vec{G}_{t}=$ $i \circ \overrightarrow{H_{t}^{\prime \prime}} \circ i$ so that $\pi_{*} \mathcal{H}_{t}^{\prime \prime} i=\pi_{*} \mathcal{G}_{t}=\pi_{*} \widehat{\mathcal{F}}_{t *}^{-1} \mathcal{H}_{t *}=\widehat{S}_{t *}^{-1} \pi_{*} \widehat{\mathcal{H}}_{t *}$. Since $i L=L$, from Lemma 2.2 we finally get the claim.

\subsection{Existence of an extremal}

In the following lemma we prove the existence of a bang-singular-bang extremal for $\left(\mathbf{P}_{\mathbf{r}}\right)$.

Lemma 4.4. There exist $\rho>0, \varepsilon>0$ and a neighbourhood $\mathcal{O}$ of $\widehat{\mu}_{0}$ in $\left(\mathbb{R}^{n}\right)^{*}$ such that for any $r,\|r\|<\rho$ there exists a unique normal bang-singular-bang extremal par $\lambda^{r}:=\left(\mu^{r}, \xi^{r}\right)$ of $\left(\mathbf{P}_{\mathbf{r}}\right)$ with the following properties

1. $\mu^{r}(0) \in \mathcal{O}$;

2. the first switching time $\tau_{1}(r)$ is in $\left[\widehat{\tau}_{1}-\varepsilon, \widehat{\tau}_{1}+\varepsilon\right]$;

3. the second switching time $\tau_{2}(r)$ is in $\left[\widehat{\tau}_{2}-\varepsilon, \widehat{\tau}_{2}+\varepsilon\right]$;

4. the final time $T(r)$ is in $[\widehat{T}-\varepsilon, \widehat{T}+\varepsilon]$.

5. the times $\tau_{1}(r), \tau_{2}(r)$ and $T(r)$ and the initial adjoint covector $\omega(r):=\mu^{r}(0)$ depend smoothly on $r$.

Moreover the bang arcs are regular

$$
u_{1} F_{1}^{r} \circ \lambda^{r}(t)>0 \quad \forall t \in\left[0, \tau_{1}^{r}\right), \quad u_{2} F_{1}^{r} \circ \lambda^{r}(t)>0 \quad \forall t \in\left(\tau_{2}^{r}, \widehat{T}\right],
$$

and the singular arc is of the first kind

$$
F_{101}^{r} \circ \lambda^{r}(t)>0 \quad \forall t \in\left[\tau_{1}^{r}, \tau_{2}^{r}\right] .
$$


Proof. The proof of the lemma is a straightforward application of the implicit function theorem. Let $B(0, \rho)$ be the ball of radius $\rho>0$ centred at the origin in $\mathbb{R}^{m}$. If $\rho$ and the neighbourhood $\mathcal{O}$ are sufficiently small, we can define the following map

$$
\begin{aligned}
& \Phi:\left(r, \omega, \tau_{1}, \tau_{2}, T\right) \in B(0, \rho) \times\left(\mathbb{R}^{n}\right)^{*} \times \mathbb{R}^{3} \mapsto \\
& \quad \pi \exp \left(T-\tau_{2}\right) \overrightarrow{H_{2}^{r}} \circ \exp \left(\tau_{2}-\tau_{1}\right) \overrightarrow{F^{S, r}} \circ \exp \tau_{1} \overrightarrow{H_{1}^{r}}\left(\omega, a^{r}\right)-b^{r} \in \mathbb{R}^{n} .
\end{aligned}
$$

Let

$$
\begin{aligned}
\Psi\left(r, \omega, \tau_{1}, \tau_{2}, T\right)=( & \Phi\left(r, \omega, \tau_{1}, \tau_{2}, T\right), F_{1}^{r} \circ \exp \tau_{1} \overrightarrow{H_{1}^{r}}\left(\omega, a^{r}\right), \\
& \left.F_{01}^{r} \circ \exp \tau_{1} \overrightarrow{H_{1}^{r}}\left(\omega, a^{r}\right), F_{0}^{r} \circ \exp \tau_{1} \overrightarrow{H_{1}^{r}}\left(\omega, a^{r}\right)-1\right),
\end{aligned}
$$

we prove that the Jacobian matrix $\left.\frac{\partial \Psi}{\partial\left(\omega, \tau_{1}, \tau_{2}, T\right)}\right|_{\left(0, \widehat{\ell}_{0}, \widehat{\tau}_{1}, \widehat{\tau}_{2}, \widehat{T}\right)}$ is non-degenerate, so that the implicit equation $\Psi\left(r, \omega, \tau_{1}, \tau_{2}, T\right)=0$ defines smooth functions

$$
\omega(r), \tau_{1}(r), \tau_{2}(r), T(r),\|r\|<\rho
$$

for some positive $\rho$. Indeed, the matrix is equal to

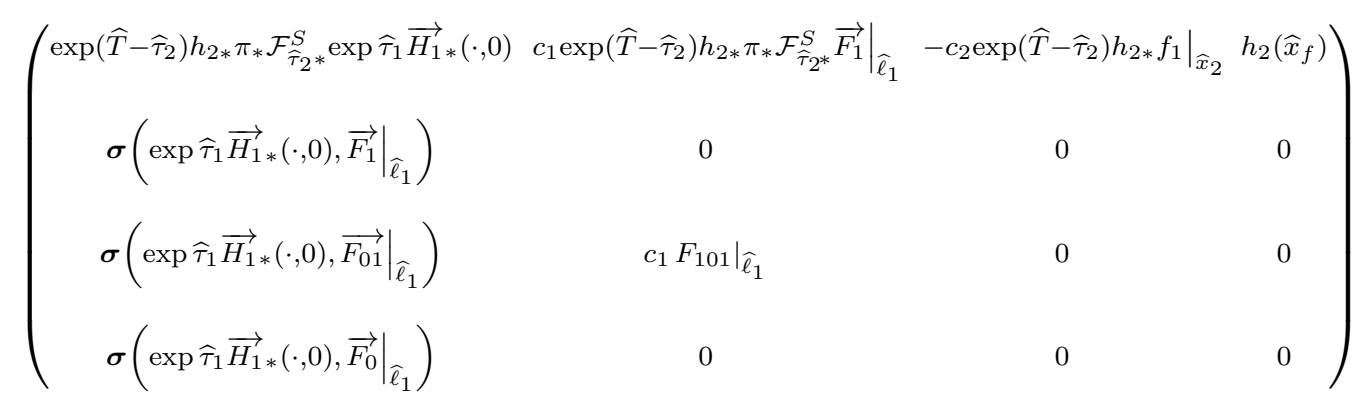

where $c_{1}:=u_{1}+\frac{F_{001}}{F_{101}}\left(\widehat{\ell}_{1}\right)$ and $c_{2}:=u_{2}+\frac{F_{001}}{F_{101}}\left(\widehat{\ell}_{2}\right)$ are nonzero (see Remark 2.2).

Since $\exp \widehat{\tau}_{1} \vec{H}_{1 *}$ is a linear isomorphism between vertical fibers, this matrix is singular if and only if there exist $\delta \ell:=(\omega, 0), \delta \tau_{1}, \delta \tau_{2}$ and $\delta T$, with at least one of them different from zero, such that

$$
\begin{aligned}
& \pi_{*} \mathcal{F}_{\widehat{\tau}_{2} *}^{S}\left(\delta \ell+\delta \tau_{1} c_{1} \vec{F}_{1}\left(\widehat{\ell}_{1}\right)\right)-\delta \tau_{2} c_{2} f_{1}\left(\widehat{x}_{2}\right)+\delta T h_{2}\left(\widehat{x}_{2}\right)=0 \\
& \boldsymbol{\sigma}\left(\delta \ell, \vec{F}_{1}\left(\widehat{\ell}_{1}\right)\right)=0 \\
& \boldsymbol{\sigma}\left(\delta \ell, \overrightarrow{F_{01}}\left(\widehat{\ell}_{1}\right)\right)+\delta \tau_{1} c_{1} F_{101}\left(\widehat{\ell}_{1}\right)=0 \\
& \boldsymbol{\sigma}\left(\delta \ell, \vec{F}_{0}\left(\widehat{\ell}_{1}\right)\right)=0
\end{aligned}
$$

Equation (26) yields $\delta \tau_{1}=\frac{-\boldsymbol{\sigma}\left(\delta \ell, \overrightarrow{\left.F_{01}\left(\widehat{\ell}_{1}\right)\right)}\right.}{c_{1} F_{101}\left(\widehat{\ell}_{1}\right)}$, hence

$$
\delta \ell_{\mathcal{S}}:=\delta \ell+\delta \tau_{1} c_{1} \overrightarrow{F_{1}}\left(\widehat{\ell}_{1}\right)=\delta \ell-\frac{\boldsymbol{\sigma}\left(\delta \ell, \overrightarrow{F_{01}}\left(\widehat{\ell}_{1}\right)\right)}{F_{101}\left(\widehat{\ell}_{1}\right)} \vec{F}_{1}\left(\widehat{\ell}_{1}\right) \in T_{\widehat{\ell}_{1}} \mathcal{S},
$$


so that by Claims 1. and 3. in Lemma 4.2

$$
\mathcal{F}_{\widehat{\tau}_{2} *}^{S}\left(\delta \ell+\delta \tau_{1} c_{1} \vec{F}_{1}\left(\widehat{\ell}_{1}\right)\right)=\widehat{\mathcal{H}}_{\widehat{\tau}_{2} *}\left(\delta \ell-\frac{\boldsymbol{\sigma}\left(\delta \ell, \overrightarrow{F_{01}}\left(\widehat{\ell}_{1}\right)\right)}{F_{101}\left(\widehat{\ell}_{1}\right)} \vec{F}_{1}\left(\widehat{\ell}_{1}\right)+a\left(\widehat{\tau}_{2}, \delta \ell_{S}\right) \vec{F}_{1}\left(\widehat{\ell}_{1}\right)\right)
$$

and equation (24) reads

$$
\begin{aligned}
\pi_{*} \mathcal{H}_{\widehat{\tau}_{2} *}(\delta \ell- & \frac{\boldsymbol{\sigma}\left(\delta \ell, \overrightarrow{F_{01}}\left(\widehat{\ell}_{1}\right)\right)}{F_{101}\left(\widehat{\ell}_{1}\right)} \vec{F}_{1}\left(\widehat{\ell}_{1}\right)+a\left(\widehat{\tau}_{2}, \delta \ell_{S}\right) \vec{F}_{1}\left(\widehat{\ell}_{1}\right)- \\
& \left.-\delta \tau_{2} c_{2} \overrightarrow{F_{1}}\left(\widehat{\ell}_{1}\right)+\delta T\left(\overrightarrow{F_{0}}+u_{2} \vec{F}_{1}\right)\left(\widehat{\ell}_{1}\right)\right)=0
\end{aligned}
$$

Equations (25) and (27) yield $\delta \ell \in \operatorname{span}\left\{f_{0}\left(\widehat{x}_{1}\right), f_{1}\left(\widehat{x}_{1}\right)\right\}^{\perp} \times\{0\} \subset L$. Thus Corollary 4.3 and equation (28) yield

$$
\delta \ell-\left(\frac{\boldsymbol{\sigma}\left(\delta \ell, \overrightarrow{F_{01}}\left(\widehat{\ell}_{1}\right)\right)}{F_{101}\left(\widehat{\ell}_{1}\right)}-a\left(\widehat{\tau}_{2}, \delta \ell_{S}\right)+\delta \tau_{2} c_{2}-u_{2} \delta T\right) \vec{F}_{1}\left(\widehat{\ell}_{1}\right)+\delta T \overrightarrow{F_{0}}\left(\widehat{\ell}_{1}\right)=0 .
$$

Since $\delta \ell, \overrightarrow{F_{0}}\left(\widehat{\ell}_{1}\right)$ and $\vec{F}_{1}\left(\widehat{\ell}_{1}\right)$ are linearly independent, equation (29) gives

$$
\delta T=0, \quad \delta \ell=\delta \ell_{S}=0, \quad \delta \tau_{2}=0 .
$$

Finally, substituting in (26), we get $\delta \tau_{1}=0$ which proves our claim, i.e.

$$
\lambda^{r}: t \mapsto \begin{cases}\exp t \overrightarrow{H_{1}^{r}}\left(\omega, a^{r}\right) & t \in\left[0, \tau_{1}(r)\right] \\ \exp \left(t-\tau_{1}(r)\right) \overrightarrow{F^{S, r}} \circ \lambda^{r}\left(\tau_{1}(r)\right) & t \in\left(\tau_{1}(r), \tau_{2}(r)\right] \\ \exp \left(T(r)-\tau_{1}(r)\right) \overrightarrow{H_{2}^{r}} \circ \lambda^{r}\left(\tau_{2}(r)\right) & t \in\left(\tau_{2}(r), T(r)\right]\end{cases}
$$

is a normal extremal for problem $\left(\mathbf{P}_{\mathbf{r}}\right)$.

By continuity, possibly restricting $\rho>0$ and $\mathcal{O}$, we can assume, for any $r \in B(0, \rho)$,

$$
\begin{aligned}
& F_{101}^{r} \circ \lambda^{r}(t)>0 \quad \forall t \in\left[\widehat{\tau}_{1}(r), \widehat{\tau}_{2}(r)\right], \\
& u_{1} F_{1}^{r} \circ \lambda^{r}(t)>0 \quad \forall t \in\left[0, \widehat{\tau}_{1}-\varepsilon\right], \\
& \left(u_{1} F_{001}^{r}+F_{101}^{r}\right) \circ \lambda^{r}(t)>0 \quad \forall t \in\left[\widehat{\tau}_{1}-\varepsilon, \widehat{\tau}_{1}+\varepsilon\right] .
\end{aligned}
$$

The Taylor expansion centered in $\tau_{1}(r)$ of the map $t \mapsto u_{1} F_{1}^{r} \circ \lambda^{r}(t)$ proves that

$$
u_{1} F_{1}^{r} \circ \lambda^{r}(t)=\frac{\left(t-\tau_{1}(r)\right)^{2}}{2}\left(u_{1} F_{001}^{r}+F_{101}^{r}\right) \circ \lambda^{r}(\theta)
$$

so that $u_{1} F_{1}^{r} \circ \lambda^{r}(t)>0$ for any $t \in\left[\widehat{\tau}_{1}-\varepsilon, \tau_{1}(r)\right)$.

Analougous proof holds for the second bang arc. 


\subsection{Proof of Theorem 3.1}

In order to prove Theorem 3.1, we need to prove the strong local optimality of the extremal pair defined in Lemma 4.4. We first prove that the extended second variation along the singular arc of $\lambda^{r}$ is coercive, for sufficiently small $\|r\|$.

Let $\tau_{1}(r)$ and $\tau_{2}(r)$ be the switching times of $\lambda^{r}$ as defined in Lemma 4.4 and let $v^{r}$ be the associated singular control, i.e.

$$
v^{r}(t):=\frac{-F_{001}^{r}}{F_{101}^{r}}\left(\lambda^{r}(t)\right) \quad t \in\left[\widehat{\tau}_{1}-\varepsilon, \widehat{\tau}_{2}+\varepsilon\right] .
$$

Following the same lines as in the nominal problem $\left(\mathbf{P}_{\mathbf{0}}\right)$, let $S^{r}{ }_{t}$ be the flow - starting at the time $\tau_{1}(r)$ - of the vector field $f_{0}^{r}+v^{r}(t) f_{1}^{r}$ and define $g_{0, t}^{r}$ and $g_{1, t}^{r}$ as the dragged vector fields at time $\tau_{1}(r)$ along such flow of the vector fields $f_{0}^{r}$ and $f_{1}^{r}$, respectively:

$$
g_{i, t}^{r}(x):=\left(S^{r}{ }_{t *}\right)^{-1} f_{i}^{r} \circ S^{r}{ }_{t}(x), \quad i=0,1 .
$$

Let $x_{i}(r):=\xi^{r}\left(\tau_{i}(r)\right), i=1,2$. Define coordinates $y^{r}$ in a neighborhood of $x_{1}^{r}$ such that

$$
y^{r}=y+O(r), \quad f_{1}^{r} \equiv \frac{\partial}{\partial y^{r}}, \quad \text { and } \quad f_{0}^{r}=\frac{\partial}{\partial y_{2}^{r}}-y_{1}^{r}\left(f_{01}^{r}\left(x_{i}(r)\right)+O\left(y^{r}\right)\right) .
$$

In such coordinates choosing $\beta^{r}\left(y^{r}\right)=-\sum_{i=2}^{n} \mu_{i}^{r} y_{i}^{r}$, where $\mu^{r}\left(\tau_{1}(r)\right)=\left(0, \mu_{2}^{r}, \ldots, \mu_{n}^{r}\right)$ the extended second variation along the singular $\operatorname{arc}$ of $\lambda^{r}$ is the quadratic form

$$
J_{\text {ext }}^{r}\left(\varepsilon_{0}, \varepsilon_{1}, w\right)=\frac{1}{2} \int_{\tau_{1}(r)}^{\tau_{2}(r)}\left(w^{2}(t) F_{101}^{r} \circ \lambda^{r}(t)+2 w(t) \zeta(t) \cdot \dot{g}_{1, t}^{r} \cdot \beta^{r}\left(\left(x_{1}(r)\right)\right) \mathrm{d} t\right.
$$

on the linear sub-space $\mathcal{W}^{r}$ of $\mathbb{R}^{2} \times L^{2}\left(\left[\tau_{1}(r), \tau_{2}(r)\right], \mathbb{R}\right)$ of the triplets $\left(\varepsilon_{0}, \varepsilon_{1}, w\right)$ such that the linear system

$$
\begin{aligned}
& \dot{\zeta}^{r}(t)=w(t) \dot{g}_{1, t}^{r}\left(x_{1}(r)\right), \\
& \zeta^{r}\left(\tau_{1}(r)\right)=\varepsilon_{0} f_{0}^{r}\left(x_{1}(r)\right)+\varepsilon_{1} f_{1}^{r}\left(x_{1}(r)\right), \quad \zeta^{r}\left(\tau_{2}(r)\right)=0 .
\end{aligned}
$$

admits a solution $\zeta^{r}$.

Lemma 4.5. Let $\lambda^{r}$ be the extremal pair of problem $\left(\mathbf{P}_{\mathbf{r}}\right)$ defined in Lemma 4.4. There exists $\rho>0$ such that for any $r,\|r\|<\rho$, the extended second variation along the singular arc of $\lambda^{r}$ is coercive.

Proof. Assume, by contradiction, there exists a sequence $r_{n} \rightarrow 0$ such that $J_{\text {ext }}^{r_{n}}$ is not coercive on $\mathcal{W}^{r_{n}}$. Define $\tau_{i}^{n}:=\tau_{i}\left(r_{n}\right), x_{i}^{n}:=x_{i}^{r_{n}}\left(\tau_{i}\left(r_{n}\right)\right), i=1,2$ and let $\varepsilon>0$ such that for any $n \in \mathbb{N},\left[\tau_{1}^{n}, \tau_{2}^{n}\right] \subset I:=\left[\widehat{\tau}_{1}-\varepsilon, \widehat{\tau}_{2}+\varepsilon\right]$. We extend any $w \in L^{2}\left(\left[\tau_{1}^{n}, \tau_{2}^{n}\right]\right)$ to the interval $I$ by prolonging it as zero and we define $H:=\mathbb{R}^{2} \times L^{2}(I, \mathbb{R})$. Then there exists $\chi^{n}=\left(\varepsilon_{0}^{n}, \varepsilon_{1}^{n}, w^{n}\right) \in H,\left\|\chi^{n}\right\|=1$ such that

$$
\varepsilon_{0}^{n} f_{0}^{r_{n}}\left(x_{1}^{n}\right)+\varepsilon_{1}^{n} f_{1}^{r_{n}}\left(x_{1}^{n}\right)+\int_{\tau_{1}^{n}}^{\tau_{2}^{n}} w^{n}(t) \dot{g}_{1, t}^{r_{n}}\left(x_{1}^{n}\right) \mathrm{d} t=0, \quad J_{\text {ext }}^{r_{n}}\left(\chi^{n}\right) \leq 0 .
$$


Without any loss of generality we can assume $\chi^{n} \rightarrow \chi^{0}=\left(\varepsilon_{0}, \varepsilon_{1}, w_{0}\right) \in H,\left\|\chi^{0}\right\| \leq 1$. Let $\zeta^{n}$ be the associated solution of system (31), for $r=r_{n}$. By standard arguments

$$
\begin{aligned}
\lim _{n \rightarrow \infty} \varepsilon_{0}^{n} f_{0}^{r_{n}}\left(x_{1}^{n}\right)+\varepsilon_{1}^{n} f_{1}^{r_{n}}\left(x_{1}^{n}\right) & +\int_{\tau_{1}^{n}}^{\tau_{2}^{n}} w^{n}(t) \dot{g}_{1, t}^{r_{n}}\left(x_{1}^{n}\right) \mathrm{d} t= \\
& =\varepsilon_{0} f_{0}\left(\widehat{x}_{1}\right)+\varepsilon_{1} f_{1}\left(\widehat{x}_{1}\right)+\int_{\widehat{\tau}_{1}}^{\widehat{\tau}_{2}} w(t) \dot{g}_{1, t}\left(\widehat{x}_{1}\right) \mathrm{d} t
\end{aligned}
$$

and

$$
\lim _{n \rightarrow \infty} \int_{\tau_{1}^{n}}^{\tau_{2}^{n}} w^{n}(t) \zeta^{n}(t) \cdot \dot{g}_{1, t}^{r_{n}}\left(x_{1}^{n}\right) \cdot \beta^{r}\left(x_{1}^{n}\right) \mathrm{d} t=\int_{\widehat{\tau}_{1}}^{\widehat{\tau}_{2}} w(t) \zeta(t) \cdot \dot{g}_{1, t}\left(\widehat{x}_{1}\right) \cdot \beta\left(\widehat{x}_{1}\right) \mathrm{d} t .
$$

Also

$$
\int_{I} w_{n}^{2}(t) R^{r_{n}}(t) \mathrm{d} t=\int_{I} w_{n}^{2}(t) R(t) \mathrm{d} t+\int_{I} w_{n}^{2}(t)\left(R^{r_{n}}-R\right)(t) \mathrm{d} t .
$$

The second addendum converges to zero since $\left\|w_{n}\right\|_{2}$ is uniformly bounded and $R^{r_{n}}$ converges to $R$ in the $L^{\infty}(I)$ norm. Let us turn to the first addendum:

$$
\begin{aligned}
& \int_{I} w_{n}^{2}(t) R(t) \mathrm{d} t=\int_{I} w_{0}^{2}(t) R(t) \mathrm{d} t+\int_{I}\left(w_{n}-w_{0}\right)^{2}(t) R(t) \mathrm{d} t+ \\
& +2 \int_{I} R(t) w_{0}(t)\left(w_{n}-w_{0}\right) \mathrm{d} t .
\end{aligned}
$$

Letting $n \rightarrow \infty$ and summing up the results in (32)-(34) we obtain

$$
\liminf _{n \rightarrow \infty} J_{\text {ext }}^{r_{n}}\left(\chi_{n}\right) \geq C\left\|\chi_{0}\right\|^{2}+\liminf _{n \rightarrow \infty} \int_{I} R(t)\left(w_{n}-w_{0}\right)^{2}(t) \mathrm{d} t
$$

If $\chi_{0}=0$ then $\left\|w_{n}\right\| \geq \frac{1}{2}$ for large enough $n$ 's so that, by (35),

$$
\liminf _{n \rightarrow \infty} J_{\text {ext }}^{r_{n}}\left(\chi_{n}\right) \geq \frac{1}{2}|I| \inf _{I} R(t)>0 .
$$

By [10] this proves the coercivity of $J_{\text {ext }}^{r_{n}}$.

If $\chi_{0} \neq 0$, then equation (35) yields the claim, provided $w_{0}(t)=0$ a.e. $t \in\left[\widehat{\tau}_{1}-\varepsilon, \widehat{\tau}_{1}\right] \cup$ $\left[\widehat{\tau}_{2}, \widehat{\tau}_{2}+\varepsilon\right]$. Since $w_{n} \rightarrow w_{0}$ in $L^{2}\left(\left[\widehat{\tau}_{1}-\varepsilon, \widehat{\tau}_{2}+\varepsilon\right]\right)$, then $w_{n} \rightarrow w_{0}$ in $L^{2}\left(\left[\widehat{\tau}_{1}-\varepsilon, \widehat{\tau}_{1}\right]\right)$.

$$
\int_{\widehat{\tau}_{1}-\varepsilon}^{\widehat{\tau}_{1}}\left|w_{n}(t)\right| \mathrm{d} t= \begin{cases}0 & \text { if } \widehat{\tau}_{1} \leq \tau_{1}^{n}, \\ \int_{\tau_{1}^{n}}^{\widehat{\tau}_{1}}\left|w_{n}(t)\right| \mathrm{d} t & \text { if } \tau_{1}^{n} \leq \widehat{\tau}_{1} .\end{cases}
$$

Since $\int_{\tau_{1}^{n}}^{\widehat{\tau}_{1}}\left|w_{n}(t)\right| \mathrm{d} t \leq\left\|w_{n}\right\|_{2} \sqrt{\widehat{\tau}_{1}-\tau_{1}^{n}} \leq \sqrt{\widehat{\tau}_{1}-\tau_{1}^{n}} \rightarrow 0$ as $n \rightarrow \infty$, we get $w_{0}(t)=0$ a.e. $t \in\left[\widehat{\tau}_{1}-\varepsilon, \widehat{\tau}_{1}\right]$. Similarly one proves $w_{0}(t)=0$ a.e. $t \in\left[\widehat{\tau}_{2}, \widehat{\tau}_{2}+\varepsilon\right]$. 
Lemma 4.5 proves (time, state)-local optimality of $\xi^{r}$, see [17]. To get state-local optimality of $\xi^{r}$ we need to prove the following:

Lemma 4.6. Under Assumptions 2.1 2.5 there exists $\rho>0$ such that for any $r,\|r\|<\rho$ the trajectory $\xi^{r}$ defined in Lemma $\overline{4.4}$ is injective.

Proof. Assume, by contradiction, there exists a sequence $\left\{r_{n}\right\}$ that converges to zero and such that there exist $0 \leq t_{1}^{n}<t_{2}^{n} \leq T\left(r_{n}\right)$ such that $\xi^{r_{n}}\left(t_{1}^{n}\right)=\xi^{r_{n}}\left(t_{2}^{n}\right)$ i.e.

$$
\int_{t_{1}^{n}}^{t_{2}^{n}}\left(f_{0}^{r_{n}}\left(\xi^{r_{n}}(s)\right)+u^{r_{n}}(s) f_{1}^{r_{n}}\left(\xi^{r_{n}}(s)\right)\right) \mathrm{d} s=0 .
$$

Up to a subsequence we can assume $t_{1}^{n} \rightarrow \bar{t}_{1}$ and $t_{2}^{n} \rightarrow \bar{t}_{2}$ as $n \rightarrow \infty$, where $0 \leq \bar{t}_{1} \leq$ $\bar{t}_{2} \leq \widehat{T}$.

If $\bar{t}_{1}<\bar{t}_{2}$, then passing to the limit in (36) we get $\widehat{\xi}\left(\bar{t}_{1}\right)=\widehat{\xi}\left(\bar{t}_{2}\right)$, a contradiction. Hence we denote as $\bar{t}$ the common value of $\bar{t}_{1}$ and $\bar{t}_{2}$.

First case: $0 \leq t_{1}^{n}<t_{2}^{n} \leq \tau_{1}\left(r_{n}\right)$.

Applying the mean value theorem componentwise in (36), for any $k=1, \ldots, n$ we get

$$
\exists s_{k}^{n} \in\left[t_{1}^{n}, t_{2}^{n}\right]:\left(h_{1}^{r_{n}}\right)_{k}\left(\xi^{r_{n}}\left(s_{k}^{n}\right)\right)=0 .
$$

Letting $n \rightarrow \infty$ in (37) we obtain $h_{1}(\widehat{\xi}(\bar{t}))=0$, a contradiction since $\bar{t} \leq \widehat{\tau}_{1}$ and $H_{1}(\widehat{\lambda}(t))=1 \quad \forall t \in\left[0, \widehat{\tau}_{1}\right]$.

Second case: $t_{1}^{n}<\tau_{1}\left(r_{n}\right)<t_{2}^{n} \leq \tau_{2}\left(r_{n}\right)$.

In this case $\bar{t}=\widehat{\tau}_{1}$ and (36) reads

$$
\int_{t_{1}^{n}}^{\tau_{1}\left(r_{n}\right)} h_{1}^{r_{n}}\left(\xi^{r_{n}}(s)\right) \mathrm{d} s=-\int_{\tau_{1}\left(r_{n}\right)}^{t_{2}^{n}}\left(f_{0}^{r_{n}}\left(\xi^{r_{n}}(s)\right)+u^{r_{n}}(s) f_{1}^{r_{n}}\left(\xi^{r_{n}}(s)\right)\right) \mathrm{d} s .
$$

Since

$$
\begin{aligned}
& \lim _{n \rightarrow \infty} \frac{1}{\tau_{1}\left(r_{n}\right)-t_{1}^{n}} \int_{t_{1}^{n}}^{\tau_{1}\left(r_{n}\right)} h_{1}^{r_{n}}\left(\xi^{r_{n}}(s)\right) \mathrm{d} s=h_{1}\left(\widehat{x}_{1}\right), \\
& \lim _{n \rightarrow \infty} \frac{1}{t_{2}^{n}-\tau_{1}\left(r_{n}\right)} \int_{\tau_{1}\left(r_{n}\right)}^{t_{2}^{n}}\left(f_{0}^{r_{n}}\left(\xi^{r_{n}}(s)\right)+u^{r_{n}}(s) f_{1}^{r_{n}}\left(\xi^{r_{n}}(s)\right)\right) \mathrm{d} s=f_{0}\left(\widehat{x}_{1}\right)+\widehat{u}\left(\widehat{\tau}_{1}+\right) f_{1}\left(\widehat{x}_{1}\right)
\end{aligned}
$$

then, by (138), the ratio $\frac{\left(t_{2}^{n}-\tau_{1}\left(r_{n}\right)\right)}{\tau_{1}\left(r_{n}\right)-t_{1}^{n}}$ converges to some quantity $L$ as $n \rightarrow \infty$ and

$$
h_{1}\left(\widehat{x}_{1}\right)=-L\left(f_{0}\left(\widehat{x}_{1}\right)+\widehat{u}\left(\widehat{\tau}_{1}+\right) f_{1}\left(\widehat{x}_{1}\right)\right)
$$

i.e.

$$
(1+L) f_{0}\left(\widehat{x}_{1}\right)+\left(1-\widehat{u}\left(\widehat{\tau}_{1}+\right)\right) f_{1}\left(\widehat{x}_{1}\right)=0,
$$

a contradiction since $f_{0}$ and $f_{1}$ are linearly independent at $\widehat{x}_{1}$ and by the discontinuity of the reference control $\widehat{u}(t)$ at time $\widehat{\tau}_{1}$, see Remark 2.1.

The other cases can be dealt with similarly. The case $t_{1}^{n} \leq \tau_{1}\left(r_{n}\right)<\tau_{2}\left(r_{n}\right) \leq t_{2}^{n}$ cannot occur since $t_{2}^{n}-t_{1}^{n} \rightarrow 0$ as $n \rightarrow \infty$ while $\tau_{2}\left(r_{n}\right)-\tau_{1}\left(r_{n}\right) \rightarrow \widehat{\tau}_{2}-\widehat{\tau}_{1}>0$. 


\subsection{Proof of Theorem 3.2}

We now give the proof of the local uniqueness result stated in Theorem 3.2 . By Assumption 2.3, there exists $\bar{\delta}>0$ such that both the maps

$$
\left.\left(u_{1} F_{001}+F_{101}\right) \circ \widehat{\lambda}\right|_{\left[\widehat{\tau}_{1}-\bar{\delta}, \widehat{\tau}_{1}\right]} \text { and }\left.\left(u_{2} F_{001}+F_{101}\right) \circ \widehat{\lambda}\right|_{\left[\widehat{\tau}_{2}, \widehat{\tau}_{2}+\bar{\delta}\right]}
$$

are strictly positive. Without any loss of generality we can assume $\bar{\delta} \in(0, \varepsilon)$, where $\varepsilon>0$ is given in Lemma 4.4. Thus the maps

$$
\left.u_{1} F_{01} \circ \hat{\lambda}\right|_{\left[\widehat{\tau}_{1}-\bar{\delta}, \widehat{\tau}_{1}\right]} \text { and }\left.u_{2} F_{01} \circ \widehat{\lambda}\right|_{\left[\widehat{\tau}_{2}, \widehat{\tau}_{2}+\bar{\delta}\right]}
$$

are strictly monotone increasing. For any $\delta \in[0, \bar{\delta}]$ set

$$
\begin{aligned}
& M_{1}(\delta)=\max \left\{\left(u_{1} F_{001}+F_{101}\right) \circ \widehat{\lambda}(t): t \in\left[\widehat{\tau}_{1}-\delta, \widehat{\tau}_{1}\right]\right\}, \\
& m_{1}(\delta)=\min \left\{\left(u_{1} F_{001}+F_{101}\right) \circ \widehat{\lambda}(t): t \in\left[\widehat{\tau}_{1}-\delta, \widehat{\tau}_{1}\right]\right\}, \\
& \alpha_{1}(\delta)=\min \left\{\left(u_{1} F_{1}\right) \circ \widehat{\lambda}(t): t \in\left[0, \widehat{\tau}_{1}-\delta\right]\right\},
\end{aligned}
$$

Then, a Taylor expansion of $u_{1} F_{1} \circ \widehat{\lambda}(t)$ in $t=\widehat{\tau}_{1}$ yields, for any $t \in\left[\widehat{\tau}_{1}-\delta, \widehat{\tau}_{1}\right]$ the inequalities

$$
\begin{aligned}
-\delta M_{1}(\delta) \leq M_{1}(\delta)\left(t-\widehat{\tau}_{1}\right) & \leq u_{1} F_{01} \circ \widehat{\lambda}(t) \leq m_{1}(\delta)\left(t-\widehat{\tau}_{1}\right), \\
\frac{m_{1}(\delta)\left(t-\widehat{\tau}_{1}\right)^{2}}{2} & \leq u_{1} F_{1} \circ \widehat{\lambda}(t) \leq \frac{M_{1}(\delta)\left(t-\widehat{\tau}_{1}\right)^{2}}{2} \leq \frac{M_{1}(\delta) \delta^{2}}{2} .
\end{aligned}
$$

Moreover without any loss of generality we can assume argmin $\left.u_{1} F_{1} \circ \widehat{\lambda}(t)\right|_{\left[0, \widehat{\tau}_{1}-\delta\right]}=\widehat{\tau}_{1}-\delta$, so that

$$
\frac{m_{1}(\delta) \delta^{2}}{2} \leq \alpha_{1}(\delta) \leq \frac{M_{1}(\delta) \delta^{2}}{2}
$$

Define

$$
\begin{aligned}
\Theta & :=\min \left\{F_{101} \circ \widehat{\lambda}(t): t \in\left[\widehat{\tau}_{1}, \widehat{\tau}_{2}\right]\right\}, \\
u_{M} & :=\sup \left\{|\widehat{u}(t)|: t \in\left(\widehat{\tau}_{1}, \widehat{\tau}_{2}\right)\right\}=\sup \left\{\left|\frac{F_{001}}{F_{101}} \circ \widehat{\lambda}(t)\right|: t \in\left(\widehat{\tau}_{1}, \widehat{\tau}_{2}\right)\right\}<1 .
\end{aligned}
$$

Similarly, set

$$
\begin{aligned}
& M_{2}(\delta)=\max \left\{\left(u_{2} F_{001}+F_{101}\right) \circ \widehat{\lambda}(t): t \in\left[\widehat{\tau}_{2}, \widehat{\tau}_{2}+\delta\right]\right\}, \\
& m_{2}(\delta)=\min \left\{\left(u_{2} F_{001}+F_{101}\right) \circ \widehat{\lambda}(t): t \in\left[\widehat{\tau}_{2}, \widehat{\tau}_{2}+\delta\right]\right\}, \\
& \alpha_{2}(\delta)=\min \left\{u_{2} F_{1} \circ \widehat{\lambda}(t): t \in\left[\widehat{\tau}_{2}+\delta, \widehat{T}\right]\right\} .
\end{aligned}
$$


Again, a Taylor expansion of $u_{2} F_{1} \circ \widehat{\lambda}(t)$ in $t=\widehat{\tau}_{2}$ yields, for any $t \in\left[\widehat{\tau}_{2}, \widehat{\tau}_{2}+\delta\right]$

$$
\begin{gathered}
m_{2}(\delta)\left(t-\widehat{\tau}_{2}\right) \leq u_{2} F_{01} \circ \widehat{\lambda}(t) \leq M_{2}(\delta)\left(t-\widehat{\tau}_{2}\right) \leq M_{2}(\delta) \delta, \\
\frac{m_{2}(\delta)\left(t-\widehat{\tau}_{2}\right)^{2}}{2} \leq u_{2} F_{1} \circ \widehat{\lambda}(t) \leq \frac{M_{2}(\delta)\left(t-\widehat{\tau}_{2}\right)^{2}}{2} \leq \frac{M_{2}(\delta) \delta^{2}}{2} .
\end{gathered}
$$

For any $\delta \in(0, \bar{\delta})$ choose $\mathcal{O}_{\delta}\left(\widehat{\ell}_{0}\right) \subset\left(\mathbb{R}^{n}\right)^{*} \times \mathbb{R}^{n}$ such that for any $\ell \in \mathcal{O}_{\delta}\left(\widehat{\ell}_{0}\right)$ the following inequalities hold:

$$
\begin{array}{lll}
u_{1} F_{1} \circ \widehat{\mathcal{F}}_{t}(\ell) \geq \frac{\alpha_{1}(\delta)}{2} & t \in\left[0, \widehat{\tau}_{1}-\delta\right] \\
\left|u_{1} F_{01} \circ \widehat{\mathcal{F}}_{t}(\ell)\right|<2 \delta M_{1}(\delta) & t \in\left[\widehat{\tau}_{1}-\delta, \widehat{\tau}_{2}+\delta\right] \\
\left|u_{1} F_{1} \circ \widehat{\mathcal{F}}_{t}(\ell)\right|<\delta^{2} M_{1}(\delta) & t \in\left[\widehat{\tau}_{1}-\delta, \widehat{\tau}_{2}+\delta\right] \\
\left|\frac{F_{001}}{F_{101}} \circ \widehat{\mathcal{F}}_{t}(\ell)\right|<\frac{1+3 u_{M}}{4} & t \in\left[\widehat{\tau}_{1}-\delta, \widehat{\tau}_{2}+\delta\right] \\
\left|F_{101} \circ \widehat{\mathcal{F}}_{t}(\ell)\right| \geq \frac{\Theta}{2} & t \in\left[\widehat{\tau}_{1}-\delta, \widehat{\tau}_{2}+\delta\right] \\
u_{2} F_{1} \circ \widehat{\mathcal{F}}_{t}(\ell) \geq \frac{\alpha_{2}(\delta)}{2} & t \in\left[\widehat{\tau}_{2}+\delta, \widehat{T}+\delta\right] .
\end{array}
$$

Set

$$
\mathcal{V}_{\delta}:=\left\{\left(t, \widehat{\mathcal{F}}_{t}(\ell)\right):(t, \ell) \in[0, \widehat{T}+\delta] \times \mathcal{O}_{\delta}\left(\widehat{\ell}_{0}\right)\right\}
$$

We choose $\rho_{\delta}>0$ such that for any $r:|r| \leq \rho_{\delta}$ the followings hold in $\mathcal{V}_{\delta}$

$$
\begin{array}{ll}
u_{1} F_{1}^{r}(\ell) \geq \frac{\alpha_{1}(\delta)}{4} & \text { if } t \leq \widehat{\tau}_{1}-\delta \\
\left|F_{01}^{r}(\ell)\right| \leq 4 \delta M_{1}(\delta) & \text { if } t \in\left[\widehat{\tau}_{1}-\delta, \widehat{\tau}_{2}+\delta\right] \\
\left|F_{1}^{r}(\ell)\right| \leq 2 \delta^{2} M_{1}(\delta) & \text { if } t \in\left[\widehat{\tau}_{1}-\delta, \widehat{\tau}_{2}+\delta\right] \\
\mid \frac{F_{001}^{r}}{F_{101}^{r}(\ell) \mid<\frac{1+u_{M}}{2}} & \text { if } t \in\left[\widehat{\tau}_{1}-\delta, \widehat{\tau}_{2}+\delta\right] \\
\left|F_{101}^{r}(\ell)\right| \geq \frac{\Theta}{4} & \text { if } t \in\left[\widehat{\tau}_{1}-\delta, \widehat{\tau}_{2}+\delta\right] \\
u_{2} F_{1}^{r}(\ell) \geq \frac{\alpha_{2}(\delta)}{4} & \text { if } t \in\left[\widehat{\tau}_{2}+\delta, \widehat{T}+\delta\right] .
\end{array}
$$

An easy consequence of (49) is

$$
\begin{aligned}
\left(F_{101}^{r} \pm F_{001}^{r}\right)(\ell) \geq F_{101}^{r}(\ell) & \left(1-\left|\frac{F_{001}^{r}}{F_{101}^{r}}(\ell)\right|\right) \geq \frac{\Theta\left(1-u_{M}\right)}{8} \\
& \text { if }(t, \ell) \in \mathcal{V}_{\delta}, \quad t \in\left[\widehat{\tau}_{1}-\delta, \widehat{\tau}_{2}+\delta\right], \quad|r|<\rho_{\delta} .
\end{aligned}
$$

Let $\tilde{\lambda}:[0, \widetilde{T}] \rightarrow\left(\mathbb{R}^{n}\right)^{*} \times \mathbb{R}^{n}$ be an extremal of $\left(\mathbf{P}_{\mathbf{r}}\right)$ such that $|\widetilde{T}-\widehat{T}|<\varepsilon$ and whose graph is in $\mathcal{V}_{\delta}$. Let $\widetilde{u}:[0, \widetilde{T}] \rightarrow[-1,1]$ be the associated control. We want to prove that $\widetilde{T}=T^{r}, \widetilde{\lambda} \equiv \lambda^{r}$ and $\widetilde{u} \equiv u^{r}$. 
The proof is split in several steps. First we prove that the trajectory of $\widetilde{\lambda}$ intersects $\Sigma^{r}$. Then we show that the entry time in $\Sigma^{r}$ is in $\left(\widehat{\tau}_{1}-\varepsilon, \widehat{\tau}_{1}+\varepsilon\right)$ and that the trajectory remains on $\Sigma^{r}$ at least untile time $\widehat{\tau}_{2}-\varepsilon$. Finally we prove that once $\widetilde{\lambda}$ has left $\Sigma^{r}$, it remains bang till the final time $\widetilde{T}$.

Step 1: $F_{1}^{r} \circ \widetilde{\lambda}(t)$ annihilates for some $t \in[0, \widetilde{T}]$.

Assume by contradiction that $F_{1}^{r} \circ \widetilde{\lambda}(t)$ never annihilates. Since $\widetilde{\lambda}(0)$ is close to $\widehat{\ell}_{0}$, we must have $\widetilde{u}(t) \equiv u_{1}$ for any $t \in[0, \widetilde{T}]$. Thus

$$
\begin{aligned}
& u_{1} F_{1}^{r} \circ \widetilde{\lambda}\left(\widehat{\tau}_{2}\right)=u_{1} F_{1}^{r} \circ \tilde{\lambda}\left(\widehat{\tau}_{1}\right)+\int_{\widehat{\tau}_{1}}^{\widehat{\tau}_{2}} u_{1} F_{01}^{r} \circ \tilde{\lambda}(s) \mathrm{d} s= \\
& =u_{1} F_{1}^{r} \circ \widetilde{\lambda}\left(\widehat{\tau}_{1}\right)+\int_{\widehat{\tau}_{1}}^{\widehat{\tau}_{2}}\left(u_{1} F_{01}^{r} \circ \widetilde{\lambda}\left(\widehat{\tau}_{1}\right)+\int_{\widehat{\tau}_{1}}^{s}\left(F_{101}^{r}+u_{1} F_{001}^{r}\right) \circ \widetilde{\lambda}(a) \mathrm{d} a\right) \mathrm{d} s \geq \\
& \geq u_{1} F_{1}^{r} \circ \tilde{\lambda}\left(\widehat{\tau}_{1}\right)+\left(\widehat{\tau}_{2}-\widehat{\tau}_{1}\right) u_{1} F_{01}^{r} \circ \widetilde{\lambda}\left(\widehat{\tau}_{1}\right)+\frac{\Theta\left(1-u_{M}\right)\left(\widehat{\tau}_{2}-\widehat{\tau}_{1}\right)^{2}}{16} \geq \\
& \geq-\left(\widehat{\tau}_{2}-\widehat{\tau}_{1}\right) 4 \delta M_{1}(\delta)+\frac{\Theta\left(1-u_{M}\right)\left(\widehat{\tau}_{2}-\widehat{\tau}_{1}\right)^{2}}{16}>2 \delta^{2} M_{1}(\delta),
\end{aligned}
$$

if $\delta$ is choosen small enough. A contradiction of (49). Define

$$
\widetilde{\tau}_{1}:=\inf \left\{t \in[0, \widetilde{T}]: F_{1}^{r} \circ \widetilde{\lambda}(t)=0\right\}
$$

so that

$$
\widetilde{\lambda}(t)=\exp t \overrightarrow{H_{1}^{r}}(\widetilde{\lambda}(0)) \quad \forall t \in\left[0, \widetilde{\tau}_{1}\right], \quad F_{1}^{r} \circ \widetilde{\lambda}\left(\widetilde{\tau}_{1}\right)=0, \quad u_{1} F_{01}^{r} \circ \widetilde{\lambda}\left(\widetilde{\tau}_{1}\right) \leq 0 .
$$

Step 2: $\widetilde{\tau}_{1} \in\left(\widehat{\tau}_{1}-\varepsilon, \widehat{\tau}_{1}+\varepsilon\right), \varepsilon$ defined in Lemma 4.4.

By definition of $\mathcal{V}_{\delta}, u_{1} F_{1}^{r} \circ \widetilde{\lambda}(t) \geq \frac{\alpha_{1}(\delta)}{4}$ if $t \leq \widehat{\tau}_{1}-\delta$, so that $\widetilde{\tau}_{1} \geq \widehat{\tau}_{1}-\delta>\widehat{\tau}_{1}-\varepsilon$ since $0<\delta<\bar{\delta}<\varepsilon$. If $\widetilde{\tau}_{1} \leq \widehat{\tau}_{1}$ we are done. Otherwise, let $s:=\widetilde{\tau}_{1}-\widehat{\tau}_{1}>0$. A Taylor expansion in $\widetilde{\tau}_{1}$ gives

$$
\begin{aligned}
& u_{1} F_{1}^{r} \circ \widetilde{\lambda}\left(\widehat{\tau}_{1}\right)=u_{1} F_{1}^{r} \circ \widetilde{\lambda}\left(\widetilde{\tau}_{1}-s\right)= \\
& =-s u_{1} F_{01}^{r} \circ \widetilde{\lambda}\left(\widetilde{\tau}_{1}\right)+\frac{s^{2}}{2}\left(u_{1} F_{001}^{r}+F_{101}^{r}\right) \circ \widetilde{\lambda}(a), \quad \text { for some } a \in\left(\widehat{\tau}_{1}, \widetilde{\tau}_{1}\right)
\end{aligned}
$$

Hence, by (49) and (51),

$$
\begin{aligned}
s & =\frac{u_{1} F_{01}^{r} \circ \widetilde{\lambda}\left(\widetilde{\tau}_{1}\right)+\sqrt{\left(u_{1} F_{01}^{r} \circ \widetilde{\lambda}\left(\widetilde{\tau}_{1}\right)\right)^{2}+\left(\left(u_{1} F_{001}^{r}+F_{101}^{r}\right) \circ \tilde{\lambda}(a)\right) 2 u_{1} F_{1}^{r} \circ \tilde{\lambda}\left(\widehat{\tau}_{1}\right)}}{\left(u_{1} F_{001}^{r}+F_{101}^{r}\right) \circ \widetilde{\lambda}(a)} \\
& \leq \sqrt{\left(\frac{u_{1} F_{01}^{r} \circ \tilde{\lambda}\left(\widetilde{\tau}_{1}\right)}{\left(u_{1} F_{001}^{r}+F_{101}^{r}\right) \circ \widetilde{\lambda}(a)}\right)^{2}+\frac{2 u_{1} F_{1}^{r} \circ \widetilde{\lambda}\left(\widehat{\tau}_{1}\right)}{\left(u_{1} F_{001}^{r}+F_{101}^{r}\right) \circ \widetilde{\lambda}(a)}} \leq \\
& \leq \sqrt{\left(\frac{8 \delta M_{1}(\delta)}{\Theta\left(1-u_{M}\right)}\right)^{2}+\frac{16 \delta^{2} M_{1}(\delta)}{\Theta\left(1-u_{M}\right)}}=\frac{4 \delta \sqrt{M_{1}(\delta)}}{\Theta\left(1-u_{M}\right)} \sqrt{4 M_{1}(\delta)+\Theta\left(1-u_{M}\right)}
\end{aligned}
$$


Therefore $s<\varepsilon$, if $\delta$ is choosen small enough.

Step 3: $F_{1}^{r} \circ \tilde{\lambda}(t) \equiv 0$ for any $t \in\left[\widetilde{\tau}_{1}, \widehat{\tau}_{2}-\varepsilon\right]$.

Let

$$
\mathcal{A}:=\left\{t \in\left(\widetilde{\tau}_{1}, \widehat{\tau}_{2}+\delta\right): F_{1}^{r} \circ \widetilde{\lambda}(t) \neq 0\right\} .
$$

$\mathcal{A}$ is open, hence it contains at least an open interval. Let $I=\left(t_{1}, t_{2}\right) \subset \mathcal{A}$ be a maximal interval. Then $F_{1}^{r} \circ \widetilde{\lambda}\left(t_{1}\right)=0$ and the control $\widetilde{u}(t)$ is constant in $I:\left.\widetilde{u}(t)\right|_{I}=\widetilde{u}_{I}:=$ $\left.\operatorname{sgn}\left(F_{1}^{r} \circ \widetilde{\lambda}(t)\right)\right|_{I}$, so that

$$
\widetilde{\lambda}(t)=\exp \left(t-t_{1}\right)\left(\overrightarrow{F_{0}^{r}}+\widetilde{u}_{1} \overrightarrow{F_{1}^{r}}\right) \circ \widetilde{\lambda}\left(t_{1}\right) \quad \forall t \in\left[t_{1}, t_{2}\right], \quad \text { and } \widetilde{u}_{I} F_{01}^{r} \circ \widetilde{\lambda}\left(t_{1}\right) \geq 0 .
$$

For any $t \in\left[t_{1}, t_{2}\right]$ we get

$$
\begin{aligned}
& \widetilde{u}_{I} F_{1}^{r} \circ \widetilde{\lambda}(t)=\int_{t_{1}}^{t} \widetilde{u}_{I} F_{01}^{r} \circ \widetilde{\lambda}(s) \mathrm{d} s \geq \widetilde{u}_{I} F_{01}^{r} \circ \widetilde{\lambda}\left(t_{1}\right)+ \\
& +\int_{t_{1}}^{t} \mathrm{~d} s \int_{t_{1}}^{s}\left(\widetilde{u}_{I} F_{001}^{r}+F_{101}^{r}\right) \circ \widetilde{\lambda}(a) \mathrm{d} a \geq \frac{\Theta\left(1-u_{M}\right)\left(t-t_{1}\right)^{2}}{16} .
\end{aligned}
$$

Two cases may occur:

First case $I=\left(t_{1}, t_{2}\right)$ for some $t_{1}<t_{2}<\widehat{\tau}_{2}+\delta$.

In this case $F_{1}^{r} \circ \widetilde{\lambda}\left(t_{2}\right)=0$. Choosing $t=t_{2}$ in (52) we get a contradiction. This shows that if $\widetilde{\lambda}$ leaves $\Sigma^{r}$ before time $\widehat{\tau}_{2}+\delta$, then it remains out of $\Sigma^{r}$, at least until time $\widehat{\tau}_{2}+\delta$.

Second case $I=\left(t_{1}, \widehat{\tau}_{2}+\delta\right)$ for some $t_{1}<\widehat{\tau}_{2}+\delta$. We need to show that $t_{1}>\widehat{\tau}_{2}-\varepsilon$. Assume, by contradiction, that $t_{1} \leq \widehat{\tau}_{2}-\varepsilon$. Choosing $t=\widehat{\tau}_{2}$ in (52) and by choosing a small enough $\delta$ we get

$$
\widetilde{u}_{1} F_{1}^{r} \circ \widetilde{\lambda}\left(\widehat{\tau}_{2}\right) \geq \frac{\Theta\left(1-u_{M}\right) \varepsilon^{2}}{16}>2 \delta^{2} M_{1}(\delta)
$$

a contradiction. Let

$$
\widetilde{\tau}_{2}:=\max \left\{t \in\left[\widetilde{\tau}_{1}, \widetilde{T}\right]: F_{1}^{r} \circ \widetilde{\lambda}(s)=0 \quad \forall s \in\left[\widetilde{\tau}_{1}, t\right]\right\} .
$$

The two cases above prove that $\tilde{\lambda}(t) \in \Sigma^{r}$ for any $t \in\left[\widetilde{\tau}_{1}, \widehat{\tau}_{2}-\varepsilon\right]$ so that $\widetilde{\tau}_{2} \geq \widehat{\tau}_{2}-\varepsilon$. If $\widetilde{\tau}_{2} \geq \widehat{\tau}_{2}+\delta$, then $F_{1}^{r} \circ \widetilde{\lambda}\left(\widehat{\tau}_{2}+\delta\right)=0$, a contradiction by (49). Thus, $\widetilde{\tau}_{2}<\widehat{\tau}_{2}+\delta<\widehat{\tau}_{2}+\varepsilon$. Step 4: $\widetilde{\tau}_{2} \leq \widehat{\tau}_{2}+\varepsilon$ and, for any $\underset{\tau}{t} \in\left(\widetilde{\tau}_{2}, \widetilde{T}\right), \widetilde{\lambda}(t) \notin \Sigma^{r}$ and $\widetilde{u}(t) \equiv u_{2}$.

By (49) and the previous step, $F_{1}^{r} \circ \widetilde{\lambda}(t)$ is non zero for any $t \in\left(t_{2}, \widetilde{T}\right]$. Hence its sign is constant and $\left.\widetilde{u}(t)\right|_{\left(t_{2}, \widetilde{T}\right]}=\widetilde{u}_{2}:=\left.\operatorname{sgn}\left(F_{1}^{r} \circ \widetilde{\lambda}(t)\right)\right|_{\left(t_{2}, \widetilde{T}\right]}$. By (49) $u_{2} F_{1}^{r} \circ \widetilde{\lambda}(t)$ is positive, hence $\widetilde{u}_{2}=u_{2}$.

Since $\widetilde{\lambda}$ is a bang-singular-bang extremal satisfying the claims of Lemma 4.4, then $\widetilde{\lambda}=\lambda^{r}$. 


\section{References}

[1] Andrei A. Agrachev and Yuri L. Sachkov. Control Theory from the Geometric Viewpoint. Springer-Verlag, 2004.

[2] Vladimir I. Arnold. Mathematical Methods in Classical Mechanics. Springer, New York, 1980.

[3] Roberto Conti. Linear differential equations and control, volume I of Institutiones Mathematicae. Istituto Nazionale di Alta Matematica, Roma, 1976. Distributed by Academic Press Inc.

[4] B. D. Craven. Control and optimization. Chapman \& Hall, 1995.

[5] Ursula Felgenhauer. Controllability and stability for problems with bang-singularbang optimal control. Private Communication.

[6] Ursula Felgenhauer. Optimality and sensitivity for semilinear bang-bang type optimal control problems. Int. J. Appl. Math. Comput. Sci., 14(4):447-454, 2004.

[7] Ursula Felgenhauer. Structural stability investigation of bang-singular-bang optimal controls. Journal of Optimization Theory and Applications, 152:605-631, 2012. 10.1007/s10957-011-9925-0.

[8] Ursula Felgenhauer, Laura Poggiolini, and Gianna Stefani. Optimality and stability result for bang-bang optimal controls with simple and double switch behaviour. CONTROL AND CYBERNETICS, 38(4B):1305 - 1325, 2009.

[9] V. Gabasov and F.M. Kirillova. High order necessary conditions for optimality. SIAM J. Control Optimization, 10:127-188, 1972.

[10] Magnus R. Hestenes. Calculus of Variations and Optimal Control Theory. John Wiley \& Sons, New York, New York, 1966.

[11] K. Malanowski. Two-norm approach in stability and sensitivity analisys of optimization and optimal control problems. Advances in Math. Sciences and Applications, 2:397-443, 1993.

[12] K. Malanowski. Regularity of solutions in stability analisys of optimization and optimal control problems. Control and Cybernetics, 23:61-86, 1994.

[13] K. Malanowski. Stability and sensitivity analysis for optimal control problems with control-state constraints. In Dissertationes Mathematicae, volume CCCXCIV. Institute of Mathematics, Polish Academy of Sciences, 2001.

[14] Laura Poggiolini and Marco Spadini. Sufficient optimality conditions for a bangbang trajectory in a bolza problem. In Andrey Sarychev, Albert Shiryaev, Manuel Guerra, and Maria do Rosário Grossinho, editors, Mathematical Control Theory and Finance, pages 337-357. Springer Berlin Heidelberg, 2008. 10.1007/978-3-540-69532$5 \_19$. 
[15] Laura Poggiolini and Marco Spadini. Strong local optimality for a bang-bang trajectory in a mayer problem. SIAM Journal on Control and Optimization, 49(1):140$161,2011$.

[16] Laura Poggiolini and Gianna Stefani. Sufficient optimality conditions for a bangsingular extremal in the minimum time problem. Control and Cybernetics, 37(2):469 $-490,2008$.

[17] Laura Poggiolini and Gianna Stefani. Bang-singular-bang extremals: sufficient optimality conditions. Journal of Dynamical and Control Systems, 17:469-514, 2011. 10.1007/s10883-011-9127-y.

[18] Laura Poggiolini and Gianna Stefani. On the minimum time problem for dodgem car-like bang-singular extremals. In Ivan Lirkov, Svetozar Margenov, and Jerzy Wasniewski, editors, Large-Scale Scientific Computing, volume 7116 of Lecture Notes in Computer Science, pages 147-154. Springer Berlin / Heidelberg, 2012. 10.1007/9783-642-29843-1_16.

[19] Laura Poggiolini and Gianna Stefani. A case study in strong optimality and structural stability of bang-singular extremals. To appear.

[20] Gianna Stefani. Strong optimality of singular trajectories. In Fabio Ancona, Alberto Bressan, Piermarco Cannarsa, Francis Clarke, and Peter R. Wolenski, editors, Geometric Control and Nonsmooth Analysis, volume 76 of Series on Advances in Mathematics for Applied Sciences, pages 300-326, Hackensack, NJ, 2008. World Scientific Publishing Co. Pte. Ltd. pp. 361 ISBN: 978-981-277-606-8.

[21] Gianna Stefani and PierLuigi Zezza. Constrained regular LQ-control problems. SIAM J. Control Optim., 35(3):876-900, 1997.

[22] Michail I. Zelikin and Vladimir F. Borisov. Theory of Chattering Control. Systems \& Control: Foundations \& Applications. Birkhauser, Boston, Basel, Berlin, 1994. 